Proceedings of the Edinburgh Mathematical Society (2003) 46, 1-34 (C)

DOI:10.1017/S0013091500000730 Printed in the United Kingdom

\title{
FINITELY RAMIFIED GRAPH-DIRECTED FRACTALS, SPECTRAL ASYMPTOTICS AND THE MULTIDIMENSIONAL RENEWAL THEOREM
}

\author{
B. M. HAMBLY ${ }^{1}$ AND S. O. G. NYBERG ${ }^{2}$ \\ ${ }^{1}$ Mathematical Institute, University of Oxford, 24-29 St Giles', \\ Oxford OX13LB,UK (hambly@maths.ox.ac.uk) \\ ${ }^{2}$ Computas AS, Vollsveien 9, PO Box 482, 1327 Lysaker, \\ Norway (son@computas.com)
}

(Received 28 June 2000)

\begin{abstract}
We consider the class of graph-directed constructions which are connected and have the property of finite ramification. By assuming the existence of a fixed point for a certain renormalization map, it is possible to construct a Laplace operator on fractals in this class via their Dirichlet forms. Our main aim is to consider the eigenvalues of the Laplace operator and provide a formula for the spectral dimension, the exponent determining the power-law scaling in the eigenvalue counting function, and establish generic constancy for the counting-function asymptotics. In order to do this we prove an extension of the multidimensional renewal theorem. As a result we show that it is possible for the eigenvalue counting function for fractals to require a logarithmic correction to the usual power-law growth.
\end{abstract}

Keywords: fractal; Dirichlet form; counting-function asymptotics; renewal theory

AMS 2000 Mathematics subject classification: Primary 35P20; 58J50

Secondary 28A80;60K05; 31C25

\section{Introduction}

The study of the Laplace operator on fractal sets has focused primarily on fractals that are exactly self-similar and have the property of finite ramification. The class of postcritically finite self-similar sets $[\mathbf{1 3}, \mathbf{1 6}]$ captures most objects with these properties. In this framework there is a reasonable understanding of the Laplace operator, in particular its spectral properties [17] and heat kernel behaviour [12]. It is hoped that these sets will give good insight into more general fractals which may be used in the modelling of physical systems.

The class of graph-directed constructions was introduced in [25] and provides a much wider range of (slightly) more realistic models. Fractals of this type first appeared in [5] and have been called different names, including recurrent iterated function systems $[\mathbf{3}]$. They are no longer exactly self-similar but do consist of self-similar components. An example of a finitely ramified graph-directed fractal, the type of set that we consider, is 

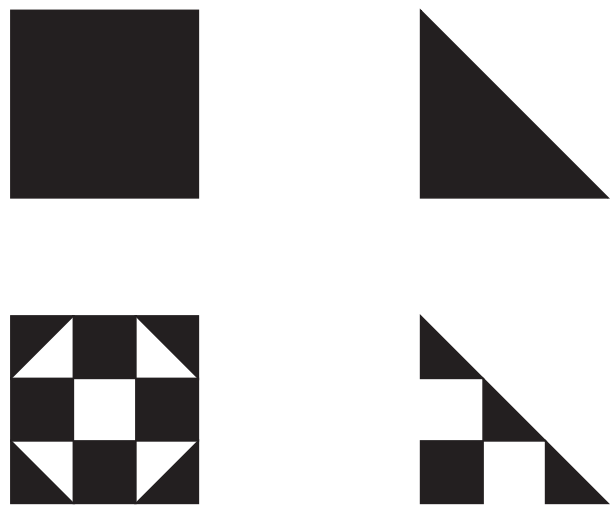

Figure 1. The two generators of a graph-directed fractal.
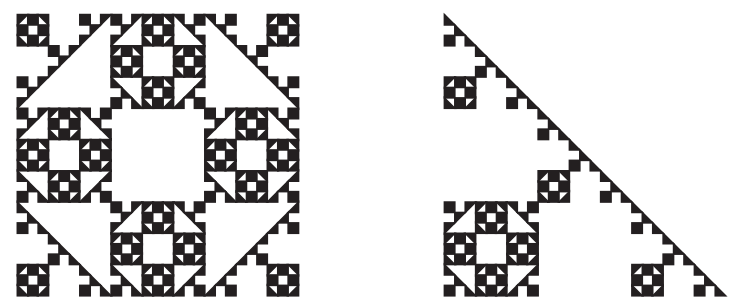

Figure 2. The vector of fractals at stage 3 .
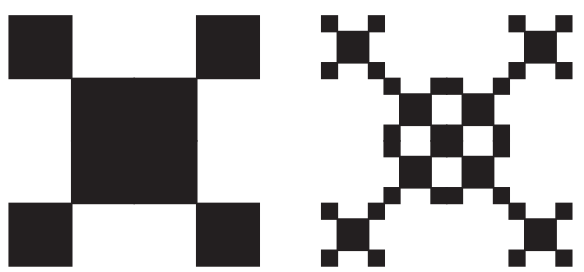

Figure 3. The first two stages of construction of a fractal with infinitely ramified generator.

shown in Figure 1. The fractal should be thought of as a pair of fractals, one based on the square and the other on the triangle. The set is formed by recursive application of the maps which replace triangles and squares with the depicted combinations of triangles and squares as shown in Figure 1. The repeated iteration of the maps leads to a pair of fractals as shown in Figure 2. In $\S 6$ we will construct a class of fractals that can be decomposed into graph-directed constructions with the finite ramification property. Two examples are the fractal in Figure 3 and the diamond fractal of [18] shown in Figure 5. Note that the generator of the fractal in Figure 3 appears infinitely ramified at stage 1 as the central square is composed of four squares which meet along edges. However, at stage 2 we see that the fractal is a finitely ramified combination of squares and rectangles.

Analysis on fractals has concentrated largely on finitely ramified fractals (a fractal is finitely ramified if any subset of the fractal can be disconnected by removing only a finite number of points) as there are very natural graph approximations to these sets. The 
construction of the Laplace operator on the fractal can be reduced to a finite-dimensional fixed-point problem for these graphs.

The framework of graph-directed constructions allows us to extend the class of fractals for which we can construct Laplace operators through quite simple extensions of the techniques developed in [13]. We will assume the existence of a solution to the canonical fixed-point problem and use this to construct a Dirichlet form on the fractal. For some discussion of this existence problem for a homogeneous random version of these fractals see [4]. This paper will then focus on the eigenvalue asymptotics of the associated Laplace operators. As these operators have compact resolvents they have discrete spectra and we can define the associated eigenvalue counting function $N(\lambda)$ as the number of eigenvalues less than $\lambda$. We will discuss the leading-order term in the asymptotic expansion of $N(\lambda)$.

There has been considerable interest in studying the behaviour of the eigenvalue counting function for various domains. In the case of a domain with fractal boundary, the WeylBerry conjecture has motivated much research. Weyl's classical result for a bounded open subset $D \subset \mathbb{R}^{n}$ states that for either the Dirichlet or Neumann Laplacian,

$$
\lim _{\lambda \rightarrow \infty} \frac{N(\lambda)}{\lambda^{n / 2}}=\frac{B_{n}|D|}{(2 \pi)^{n}}
$$

where $|D|$ denotes the $n$-dimensional volume of the set $D$ and $B_{n}$ the volume of the unit ball in $\mathbb{R}^{n}$. It was conjectured by Berry that the second-order term would be determined by the Hausdorff dimension of the boundary of $D$. Subsequent research has shown that the Minkowski dimension of the boundary of $D$ is the right notion of dimension but this does not give the precise behaviour of the second-order term in general (for a discussion see $[\mathbf{2 1}])$.

In the case where the domain itself is fractal, a number of authors have established the behaviour of the leading term. This is typically different from the Euclidean case and the power which appears in the leading term has become known as the spectral dimension, $d_{\mathrm{S}}$. In $[\mathbf{2}, \mathbf{9}-\mathbf{1 1}, \mathbf{1 5}, \mathbf{1 7}]$ it has been shown that the generic situation is the existence of a constant $C>0$, such that

$$
\lim _{\lambda \rightarrow \infty} \frac{N(\lambda)}{\lambda^{d_{\mathrm{s}} / 2}}=C
$$

However, if the scale factors in the fractal are related in a certain way, then this limit may not exist and the ratio is asymptotically a periodic function of $\log \lambda$. In the setting of symmetric fractals such as the Sierpinski gasket an explanation for this oscillation is provided by the existence of strictly localized eigenfunctions [2].

We will show in this paper that similar results hold for finitely ramified graph-directed fractals but a wider variety of behaviour is possible. If the graph defining the construction is strongly connected, then the results are given in Theorem 5.4 and are very similar to the case of post-critically finite fractals discussed in $[\mathbf{1 7}]$ with generic constancy and otherwise a possibly periodic function. If the graph is not strongly connected, then there are other possibilities; in particular, the leading-order term in the asymptotic expansion of $N(\lambda)$ may grow faster than $\lambda^{d_{s} / 2}$. Our main result is stated in Theorem 5.6 and shows 
that one may have

$$
\lim _{\lambda \rightarrow \infty} \frac{N(\lambda)}{(\log \lambda)^{m} \lambda^{d_{s} / 2}}=C^{\prime}
$$

for a constant $C^{\prime}>0$ and an integer $m \geqslant 1$.

In $[\mathbf{2 7}]$ it was shown that such a logarithmic correction term would be required when studying the spectral asymptotics of the Laplacian on a set called plain mandala. We will see that this set can be considered within the graph-directed framework and we give a more precise limit result than that of $[\mathbf{2 7}]$ in $\S 6$.

The outline of the paper is as follows. Firstly, in $\S 2$, we state and prove a multidimensional renewal theorem which will be required for proving our main results. Section 3 describes the class of finitely ramified graph-directed fractals in which we work and also uses the renewal theorem to discuss the behaviour of a natural geometric counting function. In $\S 4$ we construct Laplace operators on these fractals via their Dirichlet forms. Using some properties of the Dirichlet forms we will derive a multidimensional renewal equation for the eigenvalue counting function and use our renewal theorem in $\S 5$. In $\S 6$ we discuss some examples, including plain mandala, and construct a class of fractals whose elements may be decomposed into finitely ramified graph-directed fractals. We also discuss some other examples of fractals in this class for which we can prove the existence of a Laplace operator.

\section{The multidimensional renewal theorem}

The renewal theorem is a powerful probabilistic result that has been used in proving fine results about the behaviour of self-similar sets. Examples include obtaining Hausdorff dimension and Minkowski measurability of fractals [7], studying eigenvalue problems for domains with fractal boundary [23], and studying such problems on fractals themselves $[\mathbf{1 7}]$. A review of these ideas in the fractal context can be found in [8].

There have been several extensions of the renewal theorem to higher dimensions and we mention two that are related to the one we discuss here. Firstly, in the context of multitype age-dependent branching processes, Ryan [29] derived an extension which could be used to describe the asymptotic growth of the branching process. The second case arose in the study of self-similar measures, where Lau, Wang and Chu [22] obtained a version for separable, metrizable, locally compact, abelian groups, and used it to compute the $L^{p}$ dimension of such a measure. We will give an extension of the results of $[\mathbf{2 2}]$ in the $\mathbb{R}^{n}$ case which makes precise all the possible behaviours when the matrix of measures driving the system is reducible. An indication of the behaviour in this case was obtained in $[\mathbf{6}]$, when considering the time complexity of certain algorithms.

Firstly we need some notation. Let $M=\left[m_{i j}\right]$ be a matrix of Radon measures on $\mathbb{R}_{+}$. We will write $F$ for the matrix of distribution functions of $M$, that is $F_{i j}(t)=\int_{0}^{t} m_{i j}(\mathrm{~d} s)$ and we will write $F_{i j}(t, t+h]=F_{i j}(t+h)-F_{i j}(t)$. The indices of the matrix will be referred to as states and are the vertices of a graph $G$. The graph has a directed edge between states $i$ and $j$ if the measure $m_{i j}$ is non-zero. 
We denote the operation of convolution of a function $a$ with a measure $b$ by

$$
b * a(t)=a * b(t)=\int_{0}^{t} a(t-s) b(\mathrm{~d} s)
$$

if both $a$ and $b$ are measures, we convolve the distribution function of $a$ with the measure $b$. For two matrices $A, B$ of measures we write the $i j$ th element of $C(t)=A * B(t)$ as $c_{i j}(t)=\sum_{k} a_{i k} * b_{k j}(t)$. Let $\gamma(i, j)$ denote a directed path in the graph $G$ from vertex $i$ to vertex $j$. We define the measure $m_{\gamma(i, j)}$ by taking the convolution of the measures associated with each given edge in the path. We will also write $m_{\hat{i} i}$ for the $i$ th column of the matrix $M$ with the $i$ th element removed, similarly, $m_{i \hat{i}}$ for the $i$ th row of $M$ with the $i$ th element removed. Finally, we write the matrix of measures with both the $i$ th row and column of $M$ removed as $M_{i i}$.

We follow $[\mathbf{2 2}]$ and define the measure

$$
\nu_{1}=m_{11}+m_{1 \hat{1}} * \sum_{k=0}^{\infty}\left(M_{11}\right)^{* k} * m_{\hat{1} 1} .
$$

It is not difficult to check that, if $F(\infty)$ has maximum eigenvalue 1 and is irreducible, this is a probability measure with support given by $\cup\left\{\operatorname{supp}\left(m_{\gamma}\right): \gamma\right.$ is a simple cycle in $\left.G\right\}$. If the support is contained in a discrete subgroup of $\mathbb{R}$, we will call this measure lattice, otherwise we will call it non-lattice. By the irreducibility we see that if $\nu_{1}$ is non-lattice, then $\nu_{i}$ is non-lattice for all $i$.

We begin by stating the renewal theorem for the case of irreducible $F(\infty)$. These results appeared as Theorems 4.2 and 4.3 of [22], where they are stated with one slightly stronger condition. The proofs given in $[\mathbf{2 2}]$ use only the assumptions stated here. Firstly, we give the analogue of Blackwell's Renewal Theorem.

Theorem 2.1. We assume that $F(t)$ is a matrix of measures in which $F(\infty)$ is irreducible, has maximum eigenvalue $1, F_{i j}(0-)=0, \int_{0}^{\infty} t \mathrm{~d} F_{i j}(t)<\infty$ for all $i, j$ and for each $j$ there is at least one $i$ such that $F_{i j}(0)<F_{i j}(\infty)$. Let $V(t)=\sum_{k=0}^{\infty} F^{* k}(t)$ denote the matrix renewal measure, then if $\nu_{1}$ is non-lattice,

$$
\lim _{t \rightarrow \infty} V(t, t+h]=A h
$$

where

$$
A=\frac{\boldsymbol{u} \boldsymbol{v}^{T}}{\boldsymbol{v}^{T} \mathcal{M} \boldsymbol{u}}
$$

and $\boldsymbol{u}, \boldsymbol{v}$ are the unique normalized right and left 1-eigenvectors of $F(\infty)$ and $\mathcal{M}$ is the matrix of first moments of $F$.

If $\nu_{1}$ is lattice, with period $T$, then

$$
\lim _{t \rightarrow \infty}\left[V_{i j}\left(t+\tau_{i j}, t+\tau_{i j}+T\right)\right]=A T,
$$

for any $\tau_{i j} \in \operatorname{supp}\left(m_{\gamma(i, j)}\right)$. 
We also state a result concerning the asymptotic behaviour of the solution to the renewal equation.

Theorem 2.2. Let $\boldsymbol{z}(t)$ be directly Riemann integrable, and let $F$ be a matrix of measures satisfying the assumptions of Theorem 2.1, then the renewal equation

$$
\boldsymbol{r}(t)=\boldsymbol{z}(t)+\boldsymbol{r} * F(t)
$$

has a unique solution, bounded on finite intervals. If $\nu_{1}$ is non-lattice, then

$$
\boldsymbol{r}(t) \rightarrow \int_{0}^{\infty} \boldsymbol{z}(t) \mathrm{d} t A, \quad \text { as } t \rightarrow \infty .
$$

If $\nu_{1}$ is lattice with period $T$, then

$$
\boldsymbol{r}(t)=\lim _{n \rightarrow \infty}\left[r_{i}\left(t+\tau_{1 i}+n T\right)\right]=\sum_{k} \boldsymbol{z}(t+k T) A
$$

exists almost surely for every $t \in[0, T]$.

Remark 2.3. Note that if $\operatorname{supp} m_{\gamma(1, j)}$ has period $T$, then we can remove the vector $\tau_{1 j}$ from the periodic case. However, this is not true in general, as can be seen from [22, Remark 3.4]. From this point, whenever we encounter a multidimensional periodic function arising as a limit from a renewal equation, we will assume that the initial times were chosen in the support of the underlying measure, as in the previous two theorems.

Remark 2.4. The state 1 is used as a reference state and any other would do.

Remark 2.5. The matrix $A$ has strictly positive entries by the Perron-Frobenius Theorem.

We will concentrate on the case where $F(\infty)$ is reducible and recall some basic facts about non-negative reducible matrices. A state $i$ has access to state $j$ if there is a directed path from $i$ to $j$. The states $i$ and $j$ are said to communicate if they have access to each other. Using the communication relation, we can partition the states into equivalence classes. The spectral radius of a class is the spectral radius of $F(\infty)$ restricted to that class. A class is called basic if its spectral radius is the same as that of the matrix itself. If a class is not basic, then it is called non-basic. A class $J$ is final if $J$ has access to no other class. A chain of classes is a collection of classes such that each class has access to or from another in the collection. The length of a chain is the number of basic classes that it contains. The height of a basic class $C$ is the length of the longest chain of classes which have access to $C$. Let $S_{m}$ denote the union of basic classes of height $m+1$.

Theorem 2.6. We assume that $F(t)$ is a matrix of measures in which $F(\infty)$ has maximum eigenvalue 1 , with $F_{i j}(0-)=0, \int_{0}^{\infty} t \mathrm{~d} F_{i j}(t)<\infty$ for all $i, j$ and for each $j$ there is at least one $i$ such that $F_{i j}(0)<F_{i j}(\infty)$. Let $\boldsymbol{z}$ be a vector with components that are directly Riemann integrable functions on $\mathbb{R}_{+}$with $z_{i} \neq 0$ for all $i \in S_{0}$. If $\boldsymbol{u}$ is continuous and satisfies the renewal equation (2.2), then $\boldsymbol{u}=\boldsymbol{z} * \sum_{k=0}^{\infty} F^{* k}$ and the components $u_{i}$ satisfy: 
(1) if $i \in S_{0}$, then

$$
\lim _{t \rightarrow \infty}\left(u_{i}(t)-p_{i}(t)\right)=0,
$$

where $p_{i}$ is either periodic or constant depending on whether $\nu_{i}$ is lattice or not;

(2) if $i \in S_{m}$, for $m>0$, then

$$
\lim _{t \rightarrow \infty} t^{-m} u_{i}(t)=c_{1}(i)
$$

for some constant $c_{1}(i) \geqslant 0$;

(3) if $i \notin S=\cup_{m} S_{m}$ and there is no path from $S$ to $i$, then

$$
\lim _{t \rightarrow \infty} u_{i}(t)=0
$$

(4) if $i \notin S$ and there is a path from $S_{0}$ to $i$ but no path from $S_{l}$ for any $l>0$, then

$$
\lim _{t \rightarrow \infty}\left(u_{i}(t)-p_{i}^{\prime}(t)\right)=0,
$$

for some $p_{i}^{\prime}$ which is either constant or periodic;

(5) if $i \notin S$ and there is a path from $S_{m}$ to $i$ but no path from $S_{l}$ for any $l>m$, then

$$
\lim _{t \rightarrow \infty} t^{-m} u_{i}(t)=c_{2}(i)
$$

for some constant $c_{2}(i) \geqslant 0$.

If $i \in C_{k}$, an equivalence class in $S_{m}$, then if $z_{j} \neq 0$ for at least one $j \in C_{k}$, we have $c_{1}(i), c_{2}(i)>0$.

Before we prove this result we need some preliminary lemmas which follow from the irreducible case. Using the above notation we will write $A^{(i)}$ for the limit matrix of Theorem 2.1 corresponding to a matrix renewal measure $V^{(i)}$.

Lemma 2.7. Let $F(t)$ be a matrix of measures in which $F(\infty)$ has maximum eigenvalue 1 , with $F_{i j}(0-)=0, \int_{0}^{\infty} t \mathrm{~d} F_{i j}(t)<\infty$ for all $i, j$ and for each $j$ there is at least one $i$ such that $F_{i j}(0)<F_{i j}(\infty)$ and $F$ is irreducible. For $V=\sum_{k=0}^{\infty} F^{* k}$ and all $n \geqslant 0$ we have

$$
\lim _{t \rightarrow \infty}\left[\frac{1}{t} \int_{0}^{t}\left(\frac{t-u}{t}\right)^{n} \mathrm{~d} V_{i j}(u)\right]=\frac{1}{n+1} A .
$$

Proof. We prove this result componentwise. From Theorem 2.1 we can deduce the $n=0$ case, that is for $\epsilon>0$ there exists a $t_{0}$ such that

$$
\left|t^{-1} V_{i j}(0, t)-A_{i j}\right|<\epsilon
$$

for $t>t_{0}$ for all $i, j$. For the general case, observe that we can write

$$
\frac{1}{n+1}=t^{-n-1} \int_{0}^{t} u^{n} \mathrm{~d} u=t^{-n-1} \int_{0}^{t}(t-u)^{n} \mathrm{~d} u,
$$


and hence, using an integration by parts, we have

$$
\begin{aligned}
\left|t^{-1} \int_{0}^{t}\left(\frac{t-u}{t}\right)^{n} \mathrm{~d} V_{i j}(u)-\frac{A_{i j}}{n+1}\right| & =\left|\frac{1}{t} \int_{0}^{t}\left(\frac{t-u}{t}\right)^{n} \mathrm{~d} V_{i j}-\frac{1}{t} \int_{0}^{t}\left(\frac{t-u}{t}\right)^{n} A_{i j} \mathrm{~d} u\right| \\
& \leqslant \frac{1}{t^{n+1}} \int_{0}^{t} n u^{n-1}\left|V_{i j}(t-u)-(t-u) A_{i j}\right| \mathrm{d} u .
\end{aligned}
$$

Now use the $n=0$ case, and the fact that the measure $V_{i j}$ is bounded over finite intervals, to obtain

$$
\begin{aligned}
\left|t^{-1} \int_{0}^{t}\left(\frac{t-u}{t}\right)^{n} \mathrm{~d} V_{i j}(u)-\frac{1}{n+1} A_{i j}\right| \leqslant \frac{\epsilon}{t^{n+1}} & \int_{0}^{t-t_{0}} n u^{n-1}(t-u) \mathrm{d} u \\
& +K \frac{1}{t^{n+1}} \int_{t-t_{0}}^{t} n u^{n-1}(t-u) \mathrm{d} u,
\end{aligned}
$$

for some constant $K$. If we integrate and choose a value $t_{n}$ sufficiently large, we see that

$$
\left|t^{-1} \int_{0}^{t}\left(\frac{t-u}{t}\right)^{n} \mathrm{~d} V_{i j}(u)-\frac{1}{n+1} A_{i j}\right|<2 \epsilon, \quad t>t_{n},
$$

and hence we have the result.

Corollary 2.8. If $\boldsymbol{z}$ converges to a constant $\overline{\boldsymbol{z}}$, as $t \rightarrow \infty$, and $F$ is as above, then

$$
\lim _{t \rightarrow \infty}\left[\frac{1}{t} \int_{0}^{t}\left(\frac{t-u}{t}\right)^{n} \sum_{i} z_{i}(t-u) \mathrm{d} V_{i j}(u)\right]=\frac{1}{n+1} \bar{z} A .
$$

It is also straightforward to show the following result for finite measures.

Lemma 2.9. Let $U$ be a matrix of finite measures with $U_{i j}(0-)=0$, then for all $n \geqslant 0$ we have

$$
\lim _{t \rightarrow \infty}\left[\int_{0}^{t}\left(\frac{t-u}{t}\right)^{n} \mathrm{~d} U_{i j}(u)\right]=U(\infty) .
$$

If $\boldsymbol{z}$ converges to a constant $\overline{\boldsymbol{z}}$, as $t \rightarrow \infty$, then

$$
\lim _{t \rightarrow \infty}\left[\int_{0}^{t}\left(\frac{t-u}{t}\right)^{n} \sum_{i} z_{i}(t-u) \mathrm{d} U_{i j}(u)\right]=\bar{z} U(\infty) .
$$

A function $\boldsymbol{z}$ will be called asymptotically periodic if there exists a function $\overline{\boldsymbol{z}}$, satisfying $\overline{\boldsymbol{z}}(t)=\overline{\boldsymbol{z}}(t+k T)$ for all $t>0, k \in \mathbb{N}$, and for $\epsilon>0$, there exists a $t^{\prime}$ such that

$$
|\boldsymbol{z}(t)-\overline{\boldsymbol{z}}(t)|<\epsilon, \quad \forall t>t^{\prime} .
$$

Lemma 2.10. Let $\boldsymbol{z}$ be an asymptotically periodic function with period $T$ and let $V$ be a matrix renewal measure, then

$$
\lim _{t \rightarrow \infty} \frac{1}{t} \boldsymbol{z} * V(t)=\frac{1}{T} \int_{0}^{T} \overline{\boldsymbol{z}}(s) \mathrm{d} s A .
$$


Proof. Let $\bar{z}$ be the periodic function such that for $\epsilon>0$ there exists a $t_{0}$ such that $|\overline{\boldsymbol{z}}-\boldsymbol{z}|<\epsilon$ for $t>t_{0}$. We first show that $(1 / t)(\boldsymbol{z} * V-\overline{\boldsymbol{z}} * V) \rightarrow 0$ as $t \rightarrow \infty$. We prove this componentwise as

$$
\begin{aligned}
\left|\sum_{j} \int_{0}^{t}\left(z_{j}(t-u)-\bar{z}_{j}(t-u)\right) \mathrm{d} V_{j i}(u)\right| & \leqslant \sum_{j} \int_{0}^{t}\left|z_{j}(t-u)-\bar{z}_{j}(t-u)\right| \mathrm{d} V_{j i}(u) \\
& \leqslant \sum_{j}\left(\epsilon V_{j i}\left(t-t_{0}\right)+K V_{j i}\left(t_{0}\right)\right),
\end{aligned}
$$

where $K=\sup _{0 \leqslant u \leqslant t_{0}, i}\left|z_{i}(u)-\bar{z}_{i}(u)\right|$. Thus, dividing by $t$ and using the linear growth of $V$ from (2.3), we have the convergence.

As $\bar{z}$ is a bounded function and $V$ is uniformly bounded over finite intervals and converges to a multiple of Lebesgue measure, we have

$$
\int_{l T}^{(l+1) T} \sum_{j} \bar{z}_{j}(s+u) \mathrm{d} V_{j i}(u) \rightarrow \int_{0}^{T} \sum_{j} \bar{z}_{j}(u) A_{j i} \mathrm{~d} u, \quad \forall 0 \leqslant s \leqslant T .
$$

Now define

$$
a_{l}(t)=\frac{1}{T} \int_{l T}^{(l+1) T} \sum_{j} \bar{z}_{j}(t-u) \mathrm{d} V_{j i}(u),
$$

and observe that, by the above, for all $l$ we have

$$
a_{l}(t) \rightarrow \frac{1}{T} \int_{0}^{T} \sum_{j} \bar{z}_{j}(u) A_{j i} \mathrm{~d} u
$$

uniformly as $t \rightarrow \infty$. Finally, we can write

$$
\begin{aligned}
\frac{1}{t} \bar{z} * V(t) & =\frac{T}{t} \sum_{l=0}^{[t / T]-1} \frac{1}{T} \int_{l T}^{(l+1) T} \sum_{j} \bar{z}_{j}(t-u) \mathrm{d} V_{j i}(u)+\frac{1}{t} \int_{[t / T] T}^{t} \sum_{j} \bar{z}_{j}(t-u) \mathrm{d} V_{j i}(u) \\
& \leqslant \frac{T}{t} \sum_{l=0}^{[t / T]-1} a_{l}(t)+\frac{R_{T}}{t},
\end{aligned}
$$

where $R_{T}$ is a finite constant. As the terms in the sum converge uniformly to the claimed result, we are done.

Write $U$ for a matrix of finite measures.

Lemma 2.11. If $V^{(i)}$ is as in Lemma 2.7, $\boldsymbol{z}$ is directly Riemann integrable, and $U^{i, i+1}$ are finite matrix measures, then, for $m>1$,

$$
\begin{array}{rl}
\lim _{t \rightarrow \infty} t^{-m+1} \boldsymbol{z} & * V^{(1)} * U^{1,2} * V^{(2)} * \cdots * U^{m-1, m} * V^{(m)}(t) \\
& =\frac{1}{(m-1) !} \int_{0}^{\infty} \boldsymbol{z} * V^{(1)} * U^{1,2}(u) \mathrm{d} u A^{(2)} U^{2,3}(\infty) \cdots U^{m-1, m}(\infty) A^{(m)}
\end{array}
$$


Proof. Let

$$
I^{(m)}(t)=\boldsymbol{z} * V^{(1)} * U^{1,2} * \cdots * V^{(m)}(t) .
$$

The result for $m=1$ is the original solution to the renewal equation given in Theorem 2.2. We can write this, distinguishing between the lattice and non-lattice cases, as

$$
\lim _{t \rightarrow \infty}\left(\boldsymbol{z} * V^{(1)}(t)-p(t) A^{(1)}\right)=0,
$$

where $p(t)=\sum_{k} \boldsymbol{z}(t+k T)$ is a periodic function (it may be constant) with period $T$. We now use induction and begin by proving the result for $m=2$. First we observe that, as $U^{1,2}$ is a finite measure, $\boldsymbol{z} * V^{(1)} * U^{1,2}(t)$ is an asymptotically periodic function and we call the associated periodic function $\boldsymbol{h}$. Thus we can apply Lemma 2.10 to deduce that

$$
\frac{1}{t} \boldsymbol{z} * V^{(1)} * U^{1,2} * V^{(2)}(t) \rightarrow \frac{1}{T} \int_{0}^{T} \boldsymbol{h}(u) \mathrm{d} u A^{(2)} .
$$

This constant can then be written in terms of the asymptotically periodic part as

$$
\boldsymbol{h}(u)=\lim _{n \rightarrow \infty} \boldsymbol{z} * V^{(1)} * U^{1,2}(u+n T)=T \sum_{k} \boldsymbol{z} * V^{(1)} * U^{1,2}(u+k T),
$$

and hence

$$
\frac{1}{T} \int_{0}^{T} \boldsymbol{h}(u) \mathrm{d} u=\int_{0}^{\infty} \boldsymbol{z} * V^{(1)} * U^{1,2}(u) \mathrm{d} u .
$$

Now assume the result for $m-1$ and let the limit vector be $C^{(m-1)}$. We can write

$$
I^{(m)}(t)=I^{(m-1)} * U^{m-1, m} * V^{(m)}(t),
$$

and hence, in component form,

$$
t^{-m+1} I_{i}^{(m)}(t)=t^{-m+1} \int_{0}^{t} \sum_{j} h_{j}(t-u) \mathrm{d} V_{j i}^{(m)}(u),
$$

where

$$
h_{j}(t)=\int_{0}^{t}(t-u)^{(m-2)} \sum_{k} \frac{I_{k}^{(m-1)}(t-u)}{(t-u)^{m-2}} \mathrm{~d} U_{k j}^{m-1, m}(u) .
$$

By our inductive hypothesis and Lemma 2.9, we have

$$
t^{-m+2} h_{j}(t) \rightarrow \sum_{i} C_{i}^{(m-1)} U_{i j}^{m-1, m}(\infty),
$$

as $t \rightarrow \infty$. Thus we can rewrite (2.5) as

$$
t^{-m+1} I_{i}^{(m)}(t)=t^{-1} \int_{0}^{t}\left(\frac{t-u}{t}\right)^{m-2} \sum_{j} \frac{h_{j}(t-u)}{(t-u)^{m-2}} \mathrm{~d} V_{j i}^{(m)}(u),
$$

and apply Corollary 2.8 to show

$$
C^{(m)}=\lim _{t \rightarrow \infty} t^{-m+1} I^{(m)}(t)=\frac{1}{m-1} C^{(m-1)} U^{m-1, m}(\infty) A^{(m)} .
$$

Iterating this expression gives the result. 
Proof of Theorem 2.6. This result is an extension of that of [22]. The first part concerning the solution of the renewal equation and Parts (1), (3) and (4) of the limiting result are contained in [22, Theorem 4.5]. We only need to consider Part (2), as Part (5) will follow immediately we have Part (2).

We will write the matrix of measures in canonical form and note that if there are $k$ classes, the renewal equation can be written as

$$
\left[\boldsymbol{u}_{1}, \ldots, \boldsymbol{u}_{k}\right]=\left[\boldsymbol{z}_{1}, \ldots, \boldsymbol{z}_{k}\right]+F *\left[\boldsymbol{u}_{1}, \ldots, \boldsymbol{u}_{k}\right]
$$

where $\boldsymbol{u}_{i}, \boldsymbol{z}_{i}$ are the $\boldsymbol{u}, \boldsymbol{z}$ vectors restricted to the states in class $i$. For a non-basic class $i$, we know that $V^{(i)}$ is a finite measure.

Consider a basic class of height $m$. For the chain above this class we index the basic classes by $i_{1}, \ldots, i_{m}$ and can write down a set of renewal equations

$$
\begin{aligned}
\boldsymbol{u}_{i_{m}} & =\boldsymbol{z}_{i_{m}}+\boldsymbol{u}_{i_{m}} * F_{i_{m} i_{m}}, \\
\boldsymbol{u}_{i_{m-1}} & =\boldsymbol{z}_{i_{m-1}}+\boldsymbol{u}_{i_{m}} * U^{i_{m}, i_{m-1}}+\boldsymbol{u}_{i_{m-1}} * F_{i_{m-1} i_{m-1}}, \\
& \vdots \\
\boldsymbol{u}_{i_{1}} & =\boldsymbol{z}_{i_{1}}+\boldsymbol{u}_{i_{m}} * U^{i_{m}, i_{1}}+\cdots+\boldsymbol{u}_{i_{2}} * U^{i_{2}, i_{1}}+\boldsymbol{u}_{i_{1}} * F_{i_{1} i_{1}},
\end{aligned}
$$

where we write $U^{i_{j}, i_{j+1}}$ for the finite measure which may arise from $F_{i_{j} i_{j+1}}$ if both $i_{j}$ and $i_{j+1}$ are basic, or otherwise it is the finite measure obtained from the combination of the measures arising from intermediate non-basic classes in the chain.

We can recursively solve these separate multidimensional renewal equations. If we write $V^{\left(i_{j}\right)}=\sum_{k=0}^{\infty} F_{i_{j} i_{j}}^{* k}$, then

$$
\begin{array}{r}
\boldsymbol{u}_{i_{1}}=\left(\boldsymbol{z}_{i_{1}}+\boldsymbol{z}_{i_{2}} * V^{\left(i_{2}\right)} * U^{i_{2}, i_{1}}+\cdots+\boldsymbol{z}_{i_{m}} * V^{\left(i_{m}\right)} * U^{i_{m}, i_{m-1}} * V^{\left(i_{m-1}\right)} * \cdots\right. \\
\left.\cdots * U^{i_{3}, i_{2}} * V^{\left(i_{2}\right)} * U^{i_{2}, i_{1}}\right) * V^{\left(i_{1}\right)} .
\end{array}
$$

It is clear that each term except the first is an increasing function of $t$ and the only term that will appear in the limit is the last one. As the matrices $U^{i_{j}, i_{j-1}}$ are finite measures, we can apply Lemma 2.11 to show that there is a constant vector $c$ such that

$t^{-m+1} \boldsymbol{z}_{i_{m}} * V^{\left(i_{m}\right)} * U^{i_{m}, i_{m-1}} * V^{\left(i_{m}-1\right)} * \cdots * U^{i_{3}, i_{2}} * V^{\left(i_{2}\right)} * U^{i_{2}, i_{1}} * V^{\left(i_{1}\right)} \rightarrow \boldsymbol{c}, \quad$ as $t \rightarrow \infty$.

Finally, we must show that $c_{1}(i)>0$ if $z_{j} \neq 0$ for some $j \in C_{k}(i) \subset S_{m}$. This follows from the explicit form of the constant and the fact that the classes in the chain have access to one another, ensuring that the matrix $A^{\left(i_{m-1}\right)} U^{i_{m-1}, i_{m-2}}(\infty) \cdots U^{i_{2}, i_{1}}(\infty) A^{\left(i_{1}\right)}$ must be positive by Remark 2.5 after Theorem 2.2.

In order to apply this result to the renewal equations which occur later, we state the following corollary, a multidimensional version of that found in [16, Appendix B.4].

Corollary 2.12. Let $t^{*}>0$. Let $\boldsymbol{r}(t)$ be a vector whose components are measurable functions on $\mathbb{R}$ with $r_{i}(t)=0, \forall i$, for $t<t^{*}$, and let $\boldsymbol{z}(t)$ be a non-negative directly 
Riemann integrable function with $z_{i}(t)=0, \forall i$, for $t<t^{*}$. Assume that $\boldsymbol{r}$ satisfies the renewal equation

$$
\boldsymbol{r}(t)=\boldsymbol{z}(t)+(\boldsymbol{r} * F)(t), \quad t \in \mathbb{R} .
$$

The conclusions of Theorem 2.6 then hold.

\section{Graph-directed constructions}

In [25], graph-directed constructions were defined, extending the class of deterministic fractals. Since then this class has been studied in some detail: see, for example, [28], where fine properties of random recursive graph-directed measures were obtained. We consider here the deterministic case and consider a class of such fractals with the property of finite ramification. Note that we will work in $\mathbb{R}^{n}$ but it would be possible to define an analogue of post-critically finite (p.c.f.) self-similar sets, which are abstract metric spaces, and indexed, in this setting, by a subshift of finite type.

Let $J=\left\{J_{j}\right\}_{j \in \mathcal{S}}$ be a set of compact and connected subsets of $\mathbb{R}^{n}$, indexed by a finite set $\mathcal{S}$. Let $\mathcal{S}$ be the vertex set for a directed graph $G=(\mathcal{S}, E)$, where $E$ is the set of edges for this graph. Note that, as we will allow multiple edges, the pair $(i, j)$ does not uniquely define the edge $(i, j)$ and we write $E_{i j}$ for the set of edges from $i$ to $j$. The $k$ th edge in $E_{i j}$ may be denoted $e_{k}(i, j)$. For each $i \in \mathcal{S}$, let $E_{i}=\cup_{j \in \mathcal{S}} E_{i j}$. For each edge $e \in E$, let $\phi_{e}: \mathbb{R}^{n} \rightarrow \mathbb{R}^{n}$ be an $l_{e}^{-1}$-similitude.

In order to define a graph-directed construction we make the following assumption.

\section{Assumption 3.1.}

(1) For each $i \in \mathcal{S},\left|E_{i}\right| \geqslant 1$.

(2) For each $i \in \mathcal{S}$,

$$
\bigcup_{j \in \mathcal{S}} \bigcup_{e \in E_{i j}} \phi_{e}\left(J_{j}\right) \subset J_{i} .
$$

(3) If a path in the graph $G$ is a cycle $\left(e_{k_{1}}\left(i_{1}, i_{2}\right), \ldots, e_{k_{n-1}}\left(i_{n-1}, i_{n}\right)\right)$ with $i_{n}=i_{1}$, then

$$
\prod_{j=1}^{n-1} l_{e_{k_{j}}\left(i_{j}, i_{j+1}\right)}>1 .
$$

An object $K$ constructed from this assumption is called a geometric graph-directed construction, and $G$ is called its construction graph. We recall that a graph $G$ is connected if for each pair of vertices $i, j \in G$ there is a (non-directed) path between them. We will say that the graph $G$ is strongly connected if for each pair of vertices $i, j \in G$ there is a directed path from $i$ to $j$. A strongly connected component of $G$ is a maximal subgraph $H$ of $G$ such that $H$ is strongly connected. Strongly connected components are pairwise disjoint and do not necessarily cover $G$. A single vertex may be a strongly connected component if it loops to itself. For our construction graphs, there is at least one 
strongly connected component. We construct the fractal as the unique vector of compact sets $\left(K_{1}, \ldots, K_{|\mathcal{S}|}\right)$ which satisfies

$$
K_{i}=\bigcup_{j \in \mathcal{S}} \bigcup_{e \in E_{i j}} \phi_{e}\left(K_{j}\right)
$$

This result is given as Theorem 1 of $[\mathbf{2 5}]$ and is proved by demonstrating that the map $\psi=\bigcup_{e \in E} \phi_{e}$ is a contraction on the set of compact subsets of $J$ in the Hausdorff metric.

Let $E^{n}$ be the set of length- $n$ paths in the graph $G$. Note that $E^{n}$ is not the full set of $n$-sequences of elements of $E$, but consists of the allowable sequences $\sigma=$ $\left(e_{k_{0}}\left(i_{0}, i_{1}\right), \ldots, e_{k_{n-1}}\left(i_{n-1}, i_{n}\right)\right)$ for which $e_{k_{j}}\left(i_{j}, i_{j+1}\right) \in E$ for $j=0, \ldots, n-1$. We will write $\sigma(n)=i_{n}$ for the $n$th vertex in the path $\sigma$ where the root of the path is $\sigma(0)=i_{0}$. Let $E_{i}^{n}=\left\{\sigma \in E^{n}: \sigma(0)=i\right\}$. We further define $E^{*}=\cup_{n \in \mathbb{N}} E^{n}$. Note that $E^{\infty}$ is a Markov subshift of the full shift space generated by $\mathcal{S}$. For $\sigma \in E^{n}$, we write $\phi_{\sigma}=\phi_{\left(e_{1}, \ldots, e_{n}\right)}=\phi_{e_{1}} \circ \cdots \circ \phi_{e_{n}}$. For $\sigma \in E_{i}^{n}$, we define $K_{\sigma}=\phi_{\sigma}\left(K_{\sigma(n)}\right)$, and call such objects the $n$-cells of component $\sigma(0)$. The $J_{i}, i \in \mathcal{S}$, are 0 -cells. In this way we can define a canonical projection map from the paths to the fractal $\pi: E^{\infty} \rightarrow K$, which specifies points in component $i$ as the limit of paths in the graph starting from node $i$.

In order to determine the Hausdorff dimension of the set, we recall the open-set condition, which ensures that the overlap between sets is sufficiently small.

Definition 3.2. A graph-directed construction $K=\left\{J_{i}\right\}_{i \in \mathcal{S}}$ satisfies the open-set condition (OSC) if for all $i \in \mathcal{S}$ there are non-empty open sets $\mathcal{O}_{i} \subset \mathbb{R}^{n}$, with $\mathcal{O}_{i} \subset J_{i}$, such that $\mathcal{O}_{i} \supset \bigcup_{e \in E_{i}} \phi_{e}\left(\mathcal{O}_{j}\right)$ and $\phi_{e_{k}(i, j)}\left(\mathcal{O}_{j}\right) \cap \phi_{e_{l}(i, m)}\left(\mathcal{O}_{m}\right)=\emptyset$ for all $i, j, m \in \mathcal{S}$, $e_{k}(i, j) \in E_{i j}, e_{l}(i, m) \in E_{i m}$.

It is said to satisfy the strong open-set condition if $\mathcal{O}_{i} \cap K_{i} \neq \emptyset$ for all $i \in \mathcal{S}$.

It has been shown in [31] that if the graph is not strongly connected, then the OSC may not be equivalent to the strong open-set condition.

The Hausdorff dimension of the fractals $K_{i}$ was calculated in [25] and we give a brief discussion. Let $l_{\min }=\min _{e \in E} l_{e}$ and $l_{\max }=\max _{e \in E} l_{e}$. The length-scale factor can be defined for paths in the graph by setting

$$
l_{\sigma}=\prod_{i=1}^{n} l_{e_{i}}
$$

for permissible $\sigma=\left(e_{1}, \ldots, e_{n}\right)$. Let $\boldsymbol{H}^{s}$ be the non-negative $|\mathcal{S}| \times|\mathcal{S}|$-matrix whose entries are $h_{i j}^{s}=\sum_{e \in E_{i j}} l_{e}^{-s}$. If $s<t$, then $\left(l_{\min }\right)^{(t-s)} \boldsymbol{H}^{t} \geqslant \boldsymbol{H}^{s} \geqslant\left(l_{\max }\right)^{(t-s)} \boldsymbol{H}^{t}$ elementwise. Letting $\Phi(s)$ be the principal eigenvalue of the matrix $\boldsymbol{H}^{s}$, it is then easily seen by the above that $\Phi$ is strictly monotone decreasing and continuous in $s$. It is also easy to see that the right candidate for a self-similarity dimension would be the $t$ such that $\Phi(t)=1$. For, if we take any positive vector $\boldsymbol{x}, \lim _{n \rightarrow \infty}\left(\boldsymbol{H}^{t}\right)^{n} \boldsymbol{x}=c \boldsymbol{y}$, where $\boldsymbol{y}$ is $\boldsymbol{H}^{t}$ 's principal (right) 
eigenvector and $c$ is a constant. If, however, $s \neq t$, this limit would be either 0 or infinity. These heuristics can be formalized to give the following result, which is [25, Theorem 3]. We write $\operatorname{dim}_{H, d}(F)$ for the Hausdorff dimension of a set $F$ with respect to a metric $d$, and $\mathcal{H}^{\alpha}(F)$ for the $\alpha$-dimensional Hausdorff measure of $F$. If no metric is specified, we mean the Euclidean metric.

Theorem 3.3. If $K$ satisfies the $O S C$ and if $G$ is strongly connected, then the common Hausdorff dimension of the $K_{i}$ is given by $\operatorname{dim}_{H}\left(K_{i}\right)=\alpha=\Phi^{-1}(1)$ and $0<\mathcal{H}^{\alpha}\left(K_{i}\right)<\infty$ for all $i \in \mathcal{S}$.

In $[\mathbf{2 5}]$ the case when the graph $G$ is not strongly connected is also considered. In this situation, if we write $\operatorname{SC}(G)$ for the set of strongly connected components of the graph $G$, then the Hausdorff dimension is given by

$$
\operatorname{dim}_{H}(K)=\max \left\{\alpha_{F}: F \in \operatorname{SC}(G)\right\} .
$$

The Hausdorff measure is finite if and only if $\left\{F \in \mathrm{SC}(G): \alpha=\alpha_{F}\right\}$ consists of pairwise incomparable elements.

We will be interested in a subset of these fractals consisting of those that are finitely ramified in that the cells only meet at finitely many points. Following [16] we can give an analogue of the definition of p.c.f. self-similar sets in our setting. Let $\mathcal{C}=\cup_{i, j, k \in \mathcal{S}} \cup_{e \in E_{i j}}, e^{\prime} \in E_{i k}\left(\phi_{e}\left(K_{j}\right) \cap \phi_{e^{\prime}}\left(K_{k}\right)\right)$ be the intersection points between cells. We let the critical set be $\mathcal{C}_{\pi}=\left\{\sigma \in E^{*}: \pi(\sigma) \in \mathcal{C}\right\}$ and the post-critical set be $\mathcal{P}_{\pi}=\cup_{n \geqslant 1} \cup_{\sigma \in \mathcal{C}_{\pi}} \xi_{n}(\sigma)$, where $\xi$ is the shift map. Then $F_{i}^{(0)}=\left\{\pi(\sigma): \sigma(0)=i, \sigma \in \mathcal{P}_{\pi}\right\}$ is the set of ramification points for the fractal.

We now make an assumption that guarantees that our fractals are connected and finitely ramified.

\section{Assumption 3.4.}

(1) For each $K_{i}$, for each pair of 1-cells $A, B$, there exists a sequence of 1-cells $\left\{C_{i}, i=\right.$ $0, \ldots, n\}$ such that $C_{0}=A, C_{n}=B$ and $C_{i} \cap C_{i+1} \neq \emptyset$ for $i=1, \ldots, n-1$.

(2) The $F_{i}^{(0)}$ have finite non-zero cardinality for all $i \in \mathcal{S}$.

As in [16, Proposition 1.3.5] we can show that $K_{a} \cap K_{b}=\phi_{a}\left(F_{a(n)}^{(0)}\right) \cap \phi_{b}\left(F_{b(n)}^{(0)}\right)$ for any $a \neq b \in E_{n}^{i}$ for all $i \in \mathcal{S}$. By using these ramification points we can define a sequence of graph approximations to the fractal. For $n \geqslant 1$, we define $F_{i}^{(n)}=\cup_{\sigma \in E_{i}^{n}} \phi_{\sigma}\left(F_{\sigma(n)}^{(0)}\right)$. Note that $\left\{F_{i}^{(n)}\right\}_{n \in \mathbb{N}}$ are increasing sequences. Let $F^{(n)}=\cup_{i \in \mathcal{S}} F_{i}^{(n)}$ and $F^{(\infty)}=F^{(0)} \cup$ $\cup_{\sigma \in E^{*}} \phi_{\sigma}\left(F^{(0)}\right)$.

Definition 3.5. A set $K$ satisfying the above assumptions will be called a finitely ramified graph-directed fractal set. We will abbreviate this to FRGD (fractal) set. A component $K_{i}$ of the vector $K$ will be called an FRGD fractal.

An FRGD fractal $K_{i}$ can be a member of many FRGD sets $K$. We define a map $h$ on subsets $\mathcal{S}^{\prime}$ of $\mathcal{S}$ by setting $h\left(\mathcal{S}^{\prime}\right)=\mathcal{S}^{\prime} \cup\left\{i \in \mathcal{S}: \exists e_{k}(j, i) \in E\right.$, some $\left.j \in \mathcal{S}^{\prime}\right\}$. Let $\operatorname{frgd}(i)$ 
be the smallest set containing $i$ which is closed under $h$, and let $\operatorname{FRGD}\left(K_{i}\right)=\left\{K_{j}: j \in\right.$ $\operatorname{frgd}(i)\}$. If $G$ is strongly connected, we have $\operatorname{FRGD}\left(K_{i}\right)=K, \forall K_{i} \in K$. From the finite ramification it is easy to see that an FRGD fractal will satisfy the OSC, and hence the dimension results of Theorem 3.3 hold for these fractals.

We conclude this section by discussing a geometric counting function for an FRGD fractal, using the multidimensional renewal theorem. Let $\mathcal{N}(r)$ denote the vector of numbers of sets of radius $r$ required to cover the FRGD fractal $K$. We can then state a result concerning the limiting behaviour of this function. We begin by defining a suitable matrix renewal measure. Let

$$
m_{i j}(\mathrm{~d} x)=\sum_{e \in E_{i j}} l_{e}^{-\alpha} \delta_{\log l_{e}}(\mathrm{~d} x),
$$

where $\delta_{x}$ is a Dirac measure at $x$ and, as in (2.1), we can define the measure $\nu_{i}$ using this matrix (for simplicity we assume that it is non-lattice for all $i$ ). We note that, by choice of $\alpha, F(\infty)$ has maximum eigenvalue 1 .

Theorem 3.6. For an FRGD fractal $K$, satisfying the OSC, if the measure $\nu_{i}$ is non-lattice for all $i$, then

(1) if $i \in S_{m}$, for some $m \geqslant 0$, there exist constants $c_{3}(i)>0$ such that

$$
\lim _{r \rightarrow 0} \frac{\mathcal{N}_{i}(r)}{r^{-\alpha}|\log r|^{m}}=c_{3}(i)
$$

(2) if $i \notin S$, and there is no path from $S$ to $i$,

$$
\lim _{r \rightarrow 0} \frac{\mathcal{N}_{i}(r)}{r^{-\alpha}}=0
$$

(3) if $i \notin S$ and there is a path from $S_{m}$ to $i$ and no path from $S_{l}$ to $i$ for $l>m$, there exist constants $c_{4}(i)>0$ such that

$$
\lim _{r \rightarrow 0} \frac{\mathcal{N}_{i}(r)}{r^{-\alpha}|\log r|^{m}}=c_{4}(i) .
$$

Proof. This is a straightforward application of our renewal theorem using the ideas that can be found in [8, Proposition 7.4]. We decompose the counting function according to the first stage of construction of the set to see that

$$
\mathcal{N}_{i}(r)=\sum_{j \in \mathcal{S}} \sum_{e \in E_{i j}} \mathcal{N}_{j}\left(r l_{e}\right)-a_{i}(r)
$$

where $a_{i}(r)$ is the remainder term denoting those sets which have been multiply covered. A simple change of variable, setting $r=\mathrm{e}^{-t}$, and multiplying through by $\mathrm{e}^{\alpha t}$, shows that if we define $\mathcal{N}_{i}^{\prime}(t)=\mathrm{e}^{\alpha t} \mathcal{N}_{i}\left(\mathrm{e}^{-t}\right)$ and $a_{i}^{\prime}(t)=-\mathrm{e}^{\alpha t} a_{i}\left(\mathrm{e}^{-t}\right)$, then

$$
\mathcal{N}_{i}^{\prime}(t)=a_{i}^{\prime}(t)+\sum_{j \in \mathcal{S}} \sum_{e \in E_{i j}} l_{e}^{-\alpha} \mathcal{N}_{j}^{\prime}\left(t-\log l_{e}\right), \quad t \in \mathbb{R} .
$$


Using a truncation argument, as in [8], we can transform this into a multidimensional renewal equation with respect to the matrix renewal measure $M=\left[m_{i j}\right]$ as defined in (3.1), and with $a^{\prime}$ directly Riemann integrable. This is an equation of the form considered in $\S 2$ and hence, by suitably shifting the time axis, we can apply Corollary 2.12 to get the results. The constants are strictly positive as the function $a_{i}(r)$ cannot be 0 for all $r$.

\section{Dirichlet forms for FRGD fractals}

We will now construct Dirichlet forms on our FRGD fractals. We require a measure and begin by considering any Bernoulli measure on the fractal $K$. Later we will introduce a 'natural' measure on the fractal. We will follow the approach of [13], an essential ingredient of which is to make an assumption about the existence of a suitable harmonic structure, a fixed point of a renormalization map, on the fractal. Note that we will not restrict ourselves to the analogue of Laplace operators derived from a regular harmonic structure.

For each $i \in \mathcal{S}$, let a probability measure $\mu_{e}^{\prime}$ on $E_{i}$ be given, with $\mu_{e}^{\prime}>0$ for all $e \in E_{i}$. This induces a probability measure $\mu_{i}$ on $K_{i}$, for $i \in \mathcal{S}$, where, for $\sigma=\left(e_{1}, \ldots, e_{n}\right) \in E_{i}^{n}$,

$$
\mu_{i}\left(K_{\sigma}\right)=\mu_{\sigma}^{\prime}=\prod_{k=1}^{n} \mu_{e_{k}}^{\prime} .
$$

Definition 4.1. For a set $V$, let $\ell(V)=\{f \mid V \rightarrow \mathbb{R}\}$. For $V$ a finite set, define a conductance matrix $H$ to be a symmetric linear map with $H_{i j} \geqslant 0, H_{j j}=-\sum_{k} H_{j k}$. For $u, v \in \ell(V)$, define a symmetric bilinear form $\mathcal{E}_{H}(u, v)=-u^{\mathrm{T}} H v .(V, H)$ is called a resistance network and $\mathcal{E}_{H}$ is an irreducible Dirichlet form if $\mathcal{E}_{H}(u, u) \geqslant 0$ for all $u \in \ell(V)$ with equality if and only if $u$ is constant.

By standard convention, we write $\mathcal{E}(u)$ for $\mathcal{E}(u, u)$ whenever $\mathcal{E}$ is a Dirichlet form. The Dirichlet form can as usual be recovered from knowledge of $\mathcal{E}(u)$ by the polarization identity $\mathcal{E}(u, v)=\frac{1}{4}(\mathcal{E}(u+v)-\mathcal{E}(u-v))$.

Definition 4.2. If $\left(V, H_{1}\right)$ and $\left(U, H_{2}\right)$ are two resistance networks, $U \subset V$, and

$$
\mathcal{E}_{H_{2}}(u)=\min \left\{\mathcal{E}_{H_{1}}(v): v \in \ell(V),\left.v\right|_{U}=u\right\},
$$

we say they are compatible, and write $\left(V, H_{1}\right) \geqslant\left(U, H_{2}\right)$.

Remark 4.3. If $U \subset V$ and $\left(V, H_{1}\right)$ is a known resistance network, then (4.2) uniquely defines a resistance network $\left(U, H_{2}\right)$, called the trace of $\left(V, H_{1}\right)$ on $U$.

Lemma 4.4. Let $\left(V, H_{1}\right),\left(U, H_{2}\right)$ be connected resistance networks, $\left(V, H_{1}\right) \geqslant$ $\left(U, H_{2}\right)$. Then, for each $u \in \ell(U)$, there is a unique function $v \in \ell(V)$ such that $\mathcal{E}_{H_{2}}(u, u)=\mathcal{E}_{H_{1}}(v, v)$.

For the proof of this lemma and a discussion of resistance networks in general, see [14]. 
Remark 4.5. Note that in the above, we can regard $\ell(U)$ as a subset of $\ell(V)$. Further, we see that (4.2) holds when $\ell(U) \subset \ell(V)$, and that it can be used to define a Dirichlet form $H_{2}$ on $U$ when the Dirichlet form for $\left(U, H_{1}\right)$ is given.

As an example of this, we can determine the effective resistance between two sets $X, Y \subset V$, given a resistance network $(V, H)$ with Dirichlet form $\mathcal{E}_{H}$, as

$$
R_{H}(X, Y)=\left(\min \left\{\mathcal{E}_{H}(u): u \in \ell(V),\left.u\right|_{X}=1,\left.u\right|_{Y}=0\right\}\right)^{-1} .
$$

In order to construct harmonic structures on the fractal, as in [13], we need to find a discrete Laplace operator which is the fixed point of a renormalization map. For each $i \in \mathcal{S}$ we define the resistivity of a cell $K_{e}$ in $K_{i}$ by a choice of a positive finite weight $r_{e}, e \in E_{i}$. Let $\boldsymbol{D}=\left\{D_{i}\right\}_{i \in \mathcal{S}}$, where $\left(F_{i}^{(0)}, D_{i}\right)$ is a resistance network. Letting $\mathcal{E}_{D_{i}}^{(0)}$ be the associated Dirichlet forms, we define the Dirichlet forms on $F_{i}^{(1)}$ as

$$
\mathcal{E}_{\left(r, D_{i}\right)}^{(1)}(u)=\sum_{j \in \mathcal{S}} \sum_{e \in E_{i j}} r_{e}^{-1} \cdot \mathcal{E}_{D_{j}}^{(0)}\left(u \circ \phi_{e}\right)
$$

Equation (4.3) defines a coupling mapping $\mathcal{T}^{\prime}$, parametrized by $r$, from the cone of Dirichlet forms on $F^{(0)}$ to the cone of Dirichlet forms on $F^{(1)}$. If we apply the trace map (4.2) to $\mathcal{E}_{\left(r, D_{i}\right)}^{(1)}$, with $H_{2}=F^{(0)}$, this gives us a mapping $\mathcal{T}^{\prime \prime}$ from the cone of Dirichlet forms on $F^{(1)}$ to the cone of Dirichlet forms on $F^{(0)}$.

Definition 4.6. The renormalization map is defined to be the composition of the two maps $\mathcal{T}=\mathcal{T}^{\prime \prime} \circ \mathcal{T}^{\prime}$, parametrized by $r$, which takes the cone of Dirichlet forms on $F^{(0)}$ to the cone of Dirichlet forms on $F^{(0)}$.

Assumption 4.7. In this paper, we will assume that the map $\mathcal{T}$ for the FRGD sets with a given fixed set of $r$, has an eigenform (non-degenerate projective fixed-point) $\boldsymbol{D}=\left\{D_{i}\right\}_{i \in \mathcal{S}}$, i.e. that there exists a $\lambda>0$ such that $\mathcal{T}(\boldsymbol{D})=\lambda \boldsymbol{D}$.

From now on we will assume that we have fixed $\boldsymbol{D}$ and $r$. In the final section we will provide some non-trivial examples of FRGD fractals for which a fixed point exists for some $r$. We define the conductivity of a cell $K_{e}$ in $K_{i}$ by $\rho_{e}=\lambda / r_{e}$ for $e \in E_{i}$.

We will let $\mathcal{E}_{i}^{(0)}, i \in \mathcal{S}$, be Dirichlet forms on the respective $F_{i}^{(0)}$ corresponding to this fixed point $\boldsymbol{D}$. For $\sigma \in E^{n}$, let $\rho_{\sigma}=\prod_{i=1}^{n} \rho_{e_{i}}$. For $n \in \mathbb{N}$, define

$$
\mathcal{E}_{i}^{(n)}(u)=\sum_{\sigma \in E_{i}^{n}} \rho_{\sigma} \mathcal{E}_{\sigma(n)}^{(0)}\left(\left.u\right|_{\phi_{\sigma}\left(F_{\sigma(n)}^{(0)}\right)} \circ \phi_{\sigma}\right),
$$

and let $H_{i}^{(n)}$ be the matrices defined by

$$
\mathcal{E}_{i}^{(n)}(f, g)=-f^{\mathrm{T}} H_{i}^{(n)} g .
$$

As is easily seen, $\left(F^{(n)}, H_{i}^{(n)}\right) \leqslant\left(F^{(m)}, H_{i}^{(m)}\right)$ whenever $n \leqslant m$. This ensures that the sequence of Dirichlet forms is monotone increasing and allows us to construct the limit form. 
If the harmonic structure is regular, in that $\rho_{e}>1, \forall e \in E$, the effective resistance becomes a metric and the limit form is a local regular Dirichlet form on $L^{2}(K, \mu)$ for any Borel measure $\mu$. If we do not have regularity of the harmonic structure, we need a further condition. Let $\theta_{\sigma}=\rho_{\sigma} / \mu_{\sigma}^{\prime}$. We require the following assumption on our FRGD fractals.

Assumption 4.8. $\theta_{e}>1, \forall e \in E$.

Remark 4.9. In the light of the Einstein relation (see [1]), we see that $\theta_{\sigma}$ is the time scaling factor for the approximating random walk on the cell $K_{\sigma}$.

For $m \leqslant n$, we define the harmonic extension $P_{m, n, i}: \ell\left(F_{i}^{(m)}\right) \rightarrow \ell\left(F_{i}^{(n)}\right)$ by setting $P_{m, n, i}(u)$, for $u \in \ell\left(F_{i}^{(m)}\right)$, to be $v \in \ell\left(F_{i}^{(n)}\right)$ such that $\mathcal{E}_{i}^{(m)}(u, u)=\mathcal{E}_{i}^{(n)}(v, v)$. Let $P_{m, *, i}: \ell\left(F_{i}^{(m)}\right) \rightarrow \ell\left(F_{i}^{(\infty)}\right)$ be defined by $P_{m, *, i} u(x)=P_{m, n, i} u(x)$ for some $n \in \mathbb{N}$ such that $x \in F_{i}^{(n)}$. Let $P_{m, i} u$ be the continuous extension of $P_{m, *, i} u$.

As in [13], a function which is in the image of $P_{m, n, i}$ or $P_{m, *, i}$ or $P_{m, i}$ is said to be $m$-harmonic on $K_{i}$.

We define the bilinear form $\left(\mathcal{E}_{i}, \mathcal{F}_{i}\right)$ by

$$
\mathcal{F}_{i}=\left\{u \in L^{2}\left(K_{i}, \mu_{i}\right): \sup _{n} \mathcal{E}_{i}^{(n)}(u)<\infty\right\}
$$

and

$$
\mathcal{E}_{i}(u)=\lim _{n \rightarrow \infty} \mathcal{E}_{i}^{(n)}(u), \quad \forall u \in \mathcal{F}_{i} .
$$

Note that $\mathcal{E}_{i}\left(P_{m} u, P_{m} u\right)=\mathcal{E}_{i}^{(m)}(u, u)$.

In order to prove that we have a Dirichlet form and that the corresponding Laplace operator has compact resolvent, we follow [19], which uses ideas of [20].

For a point $x \in K_{i}$ we write $D_{m}(x)$ for the points in $\psi_{\sigma \mid m}\left(K_{\sigma(m)}\right)$, where $\sigma \mid m=$ $\left(\sigma_{1}, \ldots, \sigma_{m}\right) \in E_{i}^{m}$, for all $\sigma: \pi(\sigma)=x$.

Lemma 4.10. There exists a constant $c_{5}$ such that

$$
\int_{K_{i}}\left|f(x)-f\left(x_{m}\right)\right|^{2} \mu(\mathrm{d} x) \leqslant c_{5}\left(\min _{e} \theta_{e}\right)^{-m} \mathcal{E}_{i}(f, f), \quad \forall x_{m} \in D_{m}(x), \quad f \in \mathcal{F}_{i}, \quad \forall i \in \mathcal{S} .
$$

Proof. This can be built up from results on the zeroth level following either [20] or $[\mathbf{1 9}]$. By definition of $\mathcal{E}_{i}$ we have

$$
\begin{aligned}
\mathcal{E}_{i}(f, f) & =\sup _{m} \sum_{\sigma \in E_{i}^{m}} \rho_{\sigma} \mathcal{E}_{\sigma(m)}^{(0)}\left(f \circ \phi_{\sigma}, f \circ \phi_{\sigma}\right) \\
& \geqslant \sum_{\sigma \in E_{i}^{m}} \theta_{\sigma} \sum_{x, y=\pi(\sigma, e)}(f(x)-f(y))^{2} \mu_{\sigma}^{\prime} \\
& \geqslant c \min _{\sigma \in E_{i}^{m}} \theta_{\sigma} \int \sup _{y \in D_{m}(x)}(f(x)-f(y))^{2} \mu_{m}(\mathrm{~d} x),
\end{aligned}
$$

for a constant $c>0$, where $\mu_{m}(y)$ is the piecewise constant measure defined by $\mu_{m}(y)=$ $\mu_{\sigma}^{\prime}$ for $y \in D_{m}(x)$ for all $x \in K_{i}$. We can take a weak limit of this measure to recover 
the Bernoulli measure $\mu_{i}$ on the fractal $K_{i}$. Let $x_{m}^{a}=\pi(\sigma a)$ for $\sigma \in E_{i}^{m}$ and $a$ is a path whose projection onto the fractal is a vertex. We can then extend (4.4) to $y \in D_{m+1}(x)$ by modification of the constant $c$, giving

$$
\int \sup _{y \in D_{m+1}(x)}\left(f\left(x_{m}^{a}\right)-f(y)\right)^{2} \mu(\mathrm{d} x) \leqslant c\left(\min _{\sigma \in E_{i}} \theta_{\sigma}\right)^{-m} \mathcal{E}_{i}(f, f) .
$$

For any point $x \in K_{i}$ we can find a sequence $y_{j} \in F^{(j)}$ converging to $x$ so that

$$
\begin{aligned}
\left(\int_{K_{i}}\left|f(x)-f\left(x_{m}^{a}\right)\right|^{2} \mu(\mathrm{d} x)\right)^{1 / 2} & =\left(\int_{K_{i}}\left|\sum_{j=m}^{\infty} f\left(y_{j+1}\right)-f\left(y_{j}\right)\right|^{2} \mu(\mathrm{d} x)\right)^{1 / 2} \\
& \leqslant \sum_{j=m}^{\infty}\left(\int_{K_{i}}\left|f\left(y_{j+1}\right)-f\left(y_{j}\right)\right|^{2} \mu(\mathrm{d} x)\right)^{1 / 2} \\
& \leqslant c \sum_{j=m}^{\infty} \theta_{\min }^{-j / 2} \mathcal{E}_{i}(f, f)^{1 / 2} \\
& \leqslant c_{5}^{1 / 2} \theta_{\min }^{-m / 2} \mathcal{E}_{i}(f, f)^{1 / 2}
\end{aligned}
$$

as desired.

This result shows that $\left\{P_{i, m} f\right\}_{m}$ is a Cauchy sequence in $L^{2}\left(K_{i}, \mu_{i}\right)$. Following [19] we can show that the limit is an injective map and hence that the form $\left(\mathcal{F}_{i}, \mathcal{E}_{i}\right)$ is closed in $L^{2}\left(K_{i}, \mu_{i}\right)$. We write $\mathcal{E}_{i, \lambda}(f, f)=\mathcal{E}_{i}(f, f)+\lambda\|f\|_{L^{2}\left(K_{i}, \mu_{i}\right)}^{2}$.

Lemma 4.11. The bilinear form $\left(\mathcal{F}_{i}, \mathcal{E}_{i}\right)$ has compact $\lambda$-resolvent for all $\lambda>0$, for each $i \in \mathcal{S}$.

Proof. Define $T_{i}^{m} g(x)=g\left(x_{m}^{a}\right)$. Lemma 4.10 implies that

$$
\left\|g-T_{i}^{m} g\right\|_{L^{2}\left(K_{i}, \mu_{i}\right)}^{2} \leqslant c_{5} \theta_{\min }^{-m} \mathcal{E}_{i}(g, g) .
$$

Let $I:\left(\mathcal{F}_{i}, \mathcal{E}_{i, \lambda}\right) \rightarrow L^{2}\left(K_{i}, \mu_{i}\right)$ denote the inclusion map and observe that therefore $T_{i}^{m} \rightarrow I$ in the operator norm as

$$
\left\|I-T_{i}^{m}\right\|_{\mathcal{F}_{i} \rightarrow L^{2}\left(K_{i}, \mu_{i}\right)} \leqslant c_{5} \theta_{\min }^{-m} .
$$

As the operators $T_{i}^{m}$ are compact, we have that $I$ is compact and hence its adjoint $I^{*}$ is compact. As $I^{*}$ is the $\lambda$-resolvent we have the result.

Theorem 4.12. Let $\mathcal{P}_{i}=\bigcup_{n \in \mathbb{N}} P_{n}\left(l\left(F_{i}^{(n)}\right)\right)$. Then

(1) $\mathcal{P}_{i}$ is dense in $C\left(K_{i}, \mathbb{R}\right)$ in the topology of uniform convergence and is a core for $\mathcal{F}_{i}$

(2) $\left(\mathcal{E}_{i}, \mathcal{F}_{i}\right)$ is a regular, local Dirichlet form on $L^{2}\left(K_{i}, \mu_{i}\right)$;

(3) there is a $\mu_{i}$-symmetric Hunt process $W_{i}$, which is almost surely continuous, associated with the Dirichlet form $\left(\mathcal{E}_{i}, \mathcal{F}_{i}\right)$ on $L^{2}\left(K_{i}, \mu_{i}\right)$; and 
(4) there is a self-adjoint operator $\Delta_{i}$, with compact resolvent, associated with the Dirichlet form $\left(\mathcal{E}_{i}, \mathcal{F}_{i}\right)$ on $L^{2}\left(K_{i}, \mu_{i}\right)$.

Proof. The proof follows the proof of [16, Theorem 3.4.6].

The process $W_{i}$ will be called the diffusion associated with the triplet $\left(K_{i}, \mu_{i}, \mathcal{E}_{i}\right)$. We also introduce a natural measure for the diffusion that could be called Brownian motion on an FRGD fractal. Even though we have not shown uniqueness for our process, we will call this diffusion Brownian motion and the associated generator the Laplacian. Let $\mu^{b}$ be a measure generated by setting $\mu_{e}^{b}=\rho_{e}^{-s}$. Let $M$ be the associated matrix with elements $M_{i j}=\sum_{e \in E_{i j}} \rho_{e}^{-s}$. We set $\Phi_{M}(s)$ to be the spectral radius for $M$. The Hausdorff dimension of the FRGD fractal $K$ in the effective resistance metric is given by $\operatorname{dim}_{H, R}(K)=S=\Phi_{M}^{-1}(1)$.

\section{Spectral asymptotics}

In order to calculate the spectral dimension for FRGD fractals we will prove a result about the asymptotics of the spectral counting function. This is a straightforward extension of the ideas of $[\mathbf{1 7}]$, where we use our multidimensional version of the renewal theorem.

For each component of our vector of fractals we have a Laplacian $\Delta_{i}$, on the fractal $K_{i}$, which is the infinitesimal generator of the diffusion on $K_{i}$. If we write $(\cdot, \cdot)_{\mu_{i}}$ for the natural inner product on $L^{2}\left(K_{i}, \mu_{i}\right)$, we can also obtain the Laplacian directly from the Dirichlet form by defining $\Delta_{i} f$, for suitable $f$, as $\mathcal{E}_{i}(f, g)=-\left(\Delta_{i} f, g\right)_{\mu_{i}}$ for all $g \in \mathcal{F}_{i}$. We are interested in the eigenvalues of the operators, $\Delta_{i}$, and we define the Dirichlet eigenvalue $\lambda$ with eigenfunction $u$ for this operator to be the solution to

$$
\begin{array}{ll}
\Delta_{i} u=-\lambda u, & \text { in } K_{i}, \\
u(x)=0, & x \in \partial K_{i} .
\end{array}
$$

Note that $\partial K_{i}=F_{i}^{(0)}$ is the boundary of the component $K_{i}$ and it is a finite set.

In order to define a Neumann eigenvalue for $\Delta_{i}$, we need a notion of normal derivative. Let

$$
d_{i} u(x)=\lim _{n \rightarrow \infty} \sum_{p \in N_{n}(x)}(u(x)-u(p)) H_{i}^{(n)}(x, p),
$$

where $H_{i}^{(n)}$ is the finite difference operator defined by the $n$th Dirichlet form $\mathcal{E}_{i}^{(n)}$. The Neumann eigenvalue $\lambda$ with eigenfunction $u$ for the operator $\Delta_{i}$ is then defined by

$$
\begin{aligned}
\Delta_{i} u & =-\lambda u, & & \text { in } K_{i}, \\
d_{i} u(x) & =0, & & x \in \partial K_{i} .
\end{aligned}
$$

The eigenvalue problems can be expressed in terms of the Dirichlet form as follows. In the case of the Dirichlet eigenvalue problem, we say that $u$ is an eigenfunction for $\lambda$ if $u \in \mathcal{F}_{i}^{0}=\left\{f: f \in \mathcal{F}_{i},\left.f\right|_{\partial K_{i}=0}\right\}$, and

$$
\mathcal{E}_{i}(u, v)=\lambda(u, v)_{\mu_{i}}, \quad \forall v \in \mathcal{F}_{i}^{0} .
$$


Similarly for the Neumann eigenvalues; $u$ is an eigenfunction for $\lambda$ if $u \in \mathcal{F}_{i}$, and

$$
\mathcal{E}_{i}(u, v)=\lambda(u, v)_{\mu_{i}}, \quad \forall v \in \mathcal{F}_{i} .
$$

By Theorem 4.12 the operator $\Delta_{i}$ has compact resolvent for each $i \in \mathcal{S}$ and hence there is a discrete spectrum of eigenvalues $0 \leqslant \lambda_{1}^{(i)} \leqslant \lambda_{2}^{(i)} \leqslant \cdots$, whose only accumulation point is $\infty$. This enables us to define the spectral counting functions for the Dirichlet and Neumann eigenvalues as

$$
N_{i}^{0}(x)=\#\left\{\lambda_{k}^{(i)} \leqslant x: \lambda_{k}^{(i)} \text { is a Dirichlet eigenvalue of } \Delta_{i}\right\},
$$

and

$$
N_{i}(x)=\#\left\{\lambda_{k}^{(i)} \leqslant x: \lambda_{k}^{(i)} \text { is a Neumann eigenvalue of } \Delta_{i}\right\} .
$$

These functions are non-decreasing and right continuous with $N_{i}^{0}(0)=0$ and $N_{i}(0)=1$.

Our interest is in the large- $x$ asymptotics of the counting functions and we begin with some preliminary results. Firstly there is a natural scaling in the Dirichlet form.

Lemma 5.1. For all $f, g \in \mathcal{F}_{i}$, we have

$$
\mathcal{E}_{i}(f, g)=\sum_{j \in \mathcal{S}} \sum_{e \in E_{i j}} \rho_{e} \mathcal{E}_{j}\left(f \circ \phi_{e}, g \circ \phi_{e}\right) .
$$

Proof. We prove this by considering the $n$-level form, which can be decomposed as for $f, g \in l\left(F_{i}^{(n)}\right)$ :

$$
\mathcal{E}_{i}^{(n+1)}(f, g)=\sum_{j \in \mathcal{S}} \sum_{e \in E_{i j}} \rho_{e} \mathcal{E}_{j}^{(n)}\left(f \circ \phi_{e}, g \circ \phi_{e}\right)
$$

Now let $n \rightarrow \infty$.

As a consequence of this we have

$$
\mathcal{E}_{i}(f, g)=\sum_{\sigma \in E_{i}^{n}} \rho_{\sigma} \mathcal{E}_{\sigma(n)}\left(f \circ \phi_{\sigma}, g \circ \phi_{\sigma}\right) .
$$

The key relationships for the eigenvalue counting functions are described in the following lemma.

Lemma 5.2. For all $x \geqslant 0$ and each $i \in \mathcal{S}$ we have

$$
\sum_{j \in \mathcal{S}} \sum_{e \in E_{i j}} N_{j}^{0}\left(\theta_{e}^{-1} x\right) \leqslant N_{i}^{0}(x) \leqslant N_{i}(x) \leqslant \sum_{j \in \mathcal{S}} \sum_{e \in E_{i j}} N_{j}\left(\theta_{e}^{-1} x\right),
$$

and there exists a finite constant $M$ such that

$$
N_{i}^{0}(x) \leqslant N_{i}(x) \leqslant N_{i}^{0}(x)+M, \quad \forall x, i .
$$


Proof. We begin by making a crucial observation on the decimation of the eigenfunctions. We define a new Dirichlet form

$$
\tilde{\mathcal{F}}_{i}=\left\{f: K_{i} \backslash F_{i}^{(1)} \rightarrow \mathbb{R}, f \circ \phi_{e}=f_{e}: K_{j} \backslash F_{j}^{(0)} \rightarrow \mathbb{R}, f_{e} \in \mathcal{F}_{j}, \forall e \in E_{i j}, \forall j \in \mathcal{S}\right\},
$$

and for $f, g \in \tilde{\mathcal{F}}_{i}$ we define

$$
\tilde{\mathcal{E}}_{i}(f, g)=\sum_{j \in \mathcal{S}} \sum_{e \in E_{i j}} \rho_{e} \mathcal{E}_{j}\left(f \circ \phi_{e}, g \circ \phi_{e}\right) .
$$

It is easy to see that $\mathcal{F}_{i} \subset \tilde{\mathcal{F}}_{i}$ and $\mathcal{E}_{i}=\tilde{\mathcal{E}}_{i}$ when restricted to $\mathcal{F}_{i} \times \mathcal{F}_{i}$. The form $\left(\tilde{\mathcal{E}}_{i}, \tilde{\mathcal{F}}_{i}\right)$ is a local regular Dirichlet form on $L^{2}\left(K_{i}, \mu_{i}\right)$. The associated resolvent is compact and hence the operator has a spectrum which consists of eigenvalues. Now let $u$ be an eigenfunction for the Dirichlet form $\left(\tilde{\mathcal{E}}_{i}, \tilde{\mathcal{F}}_{i}\right)$ with eigenvalue $k$, thus

$$
\tilde{\mathcal{E}}_{i}(u, v)=k(u, v)_{\mu_{i}}, \quad \forall v \in \tilde{\mathcal{F}}_{i} .
$$

If we rewrite this, using the scaling in the Dirichlet form and in the measure, then

$$
\sum_{j \in \mathcal{S}} \sum_{e \in E_{i j}} \rho_{e} \mathcal{E}_{j}\left(u \circ \phi_{e}, v \circ \phi_{e}\right)=k \sum_{j \in \mathcal{S}} \sum_{e \in E_{i j}} \mu_{e}^{\prime}\left(u \circ \phi_{e}, v \circ \phi_{e}\right)_{\mu_{j}} .
$$

Thus we have for any $h \in \mathcal{F}_{j}$,

$$
\mathcal{E}_{j}\left(u \circ \phi_{e}, h\right)=k \rho_{e}^{-1} \mu_{e}^{\prime}\left(u \circ \phi_{e}, h\right)_{\mu_{j}}, \quad \forall e \in E_{i j},
$$

so that for $e \in E_{i j}, u_{e}=u \circ \phi_{e}$ is an eigenfunction of $\left(\mathcal{E}_{j}, \mathcal{F}_{j}\right)$ with eigenvalue $\theta_{e}^{-1} k$. Clearly, if $u_{e}$ is an eigenfunction of $\left(\mathcal{E}_{j}, \mathcal{F}_{j}\right)$ with eigenvalue $\theta_{e}^{-1} k$ for each $e \in E_{i}$, then $u$, defined by

$$
u(x)= \begin{cases}u_{e}(x), & x \in \phi_{e}\left(K_{i}\right), \\ 0, & \text { otherwise },\end{cases}
$$

is an eigenfunction for $\left(\tilde{\mathcal{E}}_{i}, \tilde{\mathcal{F}}_{i}\right)$, with eigenvalue $k$.

Let $\tilde{N}_{i}$ denote the eigenvalue counting function for $\left(\tilde{\mathcal{E}}_{i}, \tilde{\mathcal{F}}_{i}\right)$. From the decimation property of the eigenfunctions we have

$$
\begin{aligned}
\tilde{N}_{i}(x) & =\#\{k: k \leqslant x\} \\
& =\sum_{e \in E_{i}} \#\left\{k \theta_{e}^{-1}: k \theta_{e}^{-1} \leqslant x \theta_{e}^{-1}\right\} \\
& =\sum_{j \in \mathcal{S}} \sum_{e \in E_{i j}} N_{j}\left(\theta_{e}^{-1} x\right) .
\end{aligned}
$$

Observe that as the domain of $\left(\tilde{\mathcal{E}}_{i}, \tilde{\mathcal{F}}_{i}\right)$ is larger than $\left(\mathcal{E}_{i}, \mathcal{F}_{i}\right)$, by a minimax argument the eigenvalues of $\left(\tilde{\mathcal{E}}_{i}, \tilde{\mathcal{F}}_{i}\right)$ must be less than those for $\left(\mathcal{E}_{i}, \mathcal{F}_{i}\right)$. Thus we have $N_{i} \leqslant \tilde{N}_{i}$ and we have the left inequality.

We now define another Dirichlet form for the Dirichlet eigenvalues. Let $\tilde{\mathcal{F}}_{i}^{0}=\{f: f \in$ $\left.\mathcal{F}_{i}^{0},\left.f\right|_{F^{(1)}}=0\right\}$ and define $\mathcal{E}_{i}^{0}=\mathcal{E}_{i}$ restricted to $\tilde{\mathcal{F}}_{i}^{0} \times \tilde{\mathcal{F}}_{i}^{0}$. Following the same reasoning 
as above we can see that if $u$ is an eigenfunction of $\left(\tilde{\mathcal{E}}_{i}^{0}, \tilde{\mathcal{F}}_{i}^{0}\right)$ with eigenvalue $k$, then for each $j \in \mathcal{S}, e \in E_{i j}$ we have that $u_{e}$ is an eigenfunction for $\left(\mathcal{E}_{j}, \mathcal{F}_{j}\right)$ with eigenvalue $\theta_{e}^{-1} k$. As before we can derive the lower bound for the Dirichlet eigenvalue counting function.

Finally, the middle inequality follows from the minimax argument as $\mathcal{F}_{0} \subset \mathcal{F}$.

The second result is a consequence of Dirichlet-Neumann bracketing and the fact that the space of harmonic functions is finite dimensional. For more on this see [17].

In order to state our main result we define the $|\mathcal{S}| \times|\mathcal{S}|$-matrix $R_{s}$ to be the one with entries given by $r_{s}(i, j)=\sum_{e \in E_{i j}} \theta_{e}^{-s}$. We then let $\Psi(s)$ denote the spectral radius of the matrix $R_{s}$.

Theorem 5.3. If the graph is strongly connected and $d_{\mathrm{s}}^{\mu} / 2$ is the solution to the equation $\Psi(s)=1$, then

$$
0<\liminf _{x \rightarrow \infty} N_{i}^{0}(x) x^{-d_{\mathrm{s}}^{\mu} / 2} \leqslant \limsup _{x \rightarrow \infty} N_{i}^{0}(x) x^{-d_{\mathrm{s}}^{\mu} / 2}<\infty,
$$

and

$$
0<\liminf _{x \rightarrow \infty} N_{i}(x) x^{-d_{\mathrm{s}}^{\mu} / 2} \leqslant \limsup _{x \rightarrow \infty} N_{i}(x) x^{-d_{\mathrm{s}}^{\mu} / 2}<\infty .
$$

Proof. As the underlying graph is strongly connected, the matrix $R_{s}$ is a primitive matrix and hence we can apply the Perron-Frobenius Theorem. There exists an eigenvector $u$ such that

$$
u_{i}=\sum_{j \in \mathcal{S}} \sum_{e \in E_{i j}} \theta_{e}^{-d_{\mathrm{s}}^{\mu} / 2} u_{j}
$$

Let $\beta_{i}(t)=\exp \left(-t d_{\mathrm{s}}^{\mu} / 2\right) N_{i}\left(\mathrm{e}^{t}\right), \beta_{i}^{0}(t)=\exp \left(-t d_{\mathrm{s}}^{\mu} / 2\right) N_{i}^{0}\left(\mathrm{e}^{t}\right)$ and rewrite the right-hand side of (5.2) in terms of the function $\beta$ as

$$
\beta_{i}(t) \leqslant \sum_{e \in E_{i}} \theta_{e}^{-d_{\mathrm{s}}^{\mu} / 2} \beta_{j}\left(t-\log \theta_{e}\right), \quad t \in \mathbb{R}
$$

By iteration of the inequality we can write this as

$$
\beta_{i}(t) \leqslant \sum_{\sigma \in E_{i}^{n}}\left(\theta_{\sigma}\right)^{-d_{\mathrm{s}}^{\mu} / 2} \beta_{\sigma(n)}\left(t-\log \theta_{\sigma}\right), \quad t \in \mathbb{R} .
$$

This will remain true if we alter our definition of $E_{i}^{n}$ to be the addresses of variable length, which ensure that $\theta_{\sigma}=\rho_{\sigma} / \mu_{\sigma}^{\prime}$ is of size $\mathrm{e}^{n}$,

$$
\hat{E}_{i}^{n}=\left\{\sigma=\left(\sigma_{1}, \ldots, \sigma_{m}\right): \theta_{\sigma} \leqslant \mathrm{e}^{n} \leqslant \theta_{\sigma,\left(i_{m}, i_{m+1}\right)}\right\} .
$$

Note that we still have $u$ as an eigenvector in that

$$
u_{i}=\sum_{\sigma \in \hat{E}_{i}^{n}} \theta_{\sigma}^{-d_{s}^{\mu} / 2} u_{j}
$$

Hence we can write

$$
\beta_{i}(t) \leqslant \sum_{\sigma \in \hat{E}_{i}^{n}}\left(\theta_{\sigma}\right)^{-d_{\mathrm{s}}^{\mu} / 2} \beta_{\sigma(m)}\left(t-\log \theta_{\sigma}\right)
$$


Let $c_{i}^{\prime}$ be such that $\beta_{i}(t) \leqslant c_{i}^{\prime}$ for $t \in\left[0, \max _{e \in E} \log \theta_{e}\right]$ and choose an $n$ such that for $\sigma \in \hat{E}_{i}^{n}$,

$$
t-\log \left(\theta_{\sigma}\right) \in\left[0, \max _{e \in E} \log \left(\theta_{e}\right)\right]
$$

In which case

$$
\begin{aligned}
\beta_{i}(t) & \leqslant \sum_{\sigma \in \hat{E}_{i}^{n}}\left(\theta_{\sigma}\right)^{-d_{\mathrm{s}}^{\mu} / 2} \beta_{\sigma(m)}\left(t-\log \theta_{\sigma}\right) \\
& \leqslant c_{i}^{\prime} \sum_{\sigma \in \hat{E}_{i}^{n}}\left(\theta_{\sigma}\right)^{-d_{\mathrm{s}}^{\mu} / 2} \\
& \leqslant \frac{c_{i}^{\prime}}{\min _{j} u_{j}} \sum_{\sigma \in \hat{E}_{i}^{n}} u_{\sigma(m)}\left(\theta_{\sigma}\right)^{-d_{\mathrm{s}}^{\mu} / 2} \\
& =\frac{c_{i}^{\prime} u_{i}}{\min _{j} u_{j}},
\end{aligned}
$$

which is independent of $n$ and hence holds for all $t>0$. Similarly for the Dirichlet eigenvalue counting function, we can obtain a lower bound by obtaining a bound over a finite interval and then iterating. This gives a bound of the same form. In order to obtain our result we now use (5.3) to see that there exist constants $c, c^{\prime}$ such that

$$
c \beta_{i}^{0}(t) \leqslant \beta_{i}(t) \leqslant c^{\prime} \beta_{i}^{0}(t), \quad \forall t>0, \quad i \in \mathcal{S} .
$$

Using this, and rewriting in terms of the eigenvalue counting function $N$, we have the result.

We can provide a finer result using our multidimensional renewal Theorem 2.6. Again we can follow $[\mathbf{1 7}]$ and write equation (5.2) as a renewal type equation.

Let

$$
\xi_{i}(t)=\beta_{i}^{0}(t)-\sum_{j \in \mathcal{S}} \sum_{e \in E_{i j}} \theta_{e}^{-d_{\mathrm{s}}^{\mu} / 2} \beta_{j}^{0}\left(t-\log \theta_{e}\right),
$$

so that $\xi$ is a non-negative bounded function of $t$. We also define the measure $m_{i j}$ to be

$$
m_{i j}(\mathrm{~d} s)=\sum_{e \in E_{i j}} \theta_{e}^{-d_{\mathrm{s}}^{\mu} / 2} \delta_{\log \theta_{e}}(\mathrm{~d} s) .
$$

Let $F(t)$ denote the matrix of measures, expressed as distribution functions. Note that by construction the maximum eigenvalue of $F(\infty)$ is 1 . We can now write

$$
\beta_{i}^{0}(t)=\xi_{i}(t)+\sum_{j \in \mathcal{S}} \int_{0}^{\infty} \beta_{j}^{0}(t-s) m_{i j}(\mathrm{~d} s), \quad t \in \mathbb{R},
$$

which can be expressed as

$$
\beta^{0}(t)=\xi(t)+\left(F * \beta^{0}\right)(t), \quad t \in \mathbb{R} .
$$


By taking the transpose, this is a matrix renewal equation of the type considered in $\S 2$. Recall that the lattice/non-lattice dichotomy depends upon the probability measure defined by

$$
\nu_{11}^{1}=m_{11}+m_{1 \hat{1}} * \sum_{k=0}^{\infty} M_{11}^{* k} * m_{\hat{1} 1} .
$$

Thus we will be in the lattice case if, for any path $\gamma$ in the graph from node 1 to itself, $\log \theta_{\gamma}$ is supported on a discrete subgroup of $\mathbb{R}$.

We are now ready to state our main results. These describe the behaviour of the leading-term asymptotics for the spectral counting function and involve a simple application of the multidimensional renewal theorem.

Theorem 5.4. For an FRGD fractal which is strongly connected, if $\nu_{11}^{1}$ is not lattice, then there exist constants $c_{6}(i), c_{7}(i)>0$ such that

$$
\lim _{x \rightarrow \infty} N_{i}(x) x^{-d_{\mathrm{s}}^{\mu} / 2}=c_{6}(i),
$$

and

$$
\lim _{x \rightarrow \infty} N_{i}^{0}(x) x^{-d_{\mathrm{s}}^{\mu} / 2}=c_{7}(i)
$$

If the measure $\nu_{11}^{1}$ is lattice, then there exist periodic functions $p_{i}, p_{i}^{\prime}$ such that

$$
\lim _{x \rightarrow \infty}\left(N_{i}(x) x^{-d_{\mathrm{s}}^{\mu} / 2}-p_{i}(\log x)\right)=0
$$

and

$$
\lim _{x \rightarrow \infty}\left(N_{i}^{0}(x) x^{-d_{\mathrm{s}}^{\mu} / 2}-p_{i}^{\prime}(\log x)\right)=0
$$

Proof. We first observe that the first Dirichlet eigenvalue $\lambda_{1}>0$. Thus there exists an $x_{0} \in \mathbb{R}$ such that for $x<x_{0}$, the counting function $N_{i}^{0}(x)=0$. Thus we can shift our counting function and, as the graph is strongly connected, apply Corollary 2.12. The case of the Neumann counting function follows from (5.3).

Remark 5.5. In the case of regular p.c.f. self-similar sets, the spectral dimension $d_{\mathrm{s}}$ can be defined as the maximal value of the spectral exponent $d_{\mathrm{s}}^{\mu}$ over all Bernoulli measures [17]. In our setting, in the corresponding regular case where $\rho_{e}>1$ for all $e \in E$, we can maximize over the product measure on the Markov subshift and define $d_{\mathrm{s}}=\max _{\mu} d_{\mathrm{s}}^{\mu}$. It is not difficult to see that the measure $\mu_{e}^{b}=\rho_{e}^{-S}$ will achieve the maximum. For this measure, $\theta_{e}=\rho_{e}^{S+1}$ and the spectral dimension satisfies $\Psi\left(d_{\mathrm{s}} / 2\right)=1$. The remark at the end of $\S 4$ allows us to write $\Psi(u)=\Phi_{M}(u(S+1))$ and hence $\Psi(u)=1$ when $u=d_{\mathrm{s}} / 2=S /(S+1)$, where $S$ is the Hausdorff dimension of the set in the effective resistance metric. This relationship between the spectral dimension and the Hausdorff dimension in the effective resistance metric has been observed for other finitely ramified fractals, such as p.c.f. fractals [16] and random recursive Sierpinski gaskets [11]. 
We now assume that the graph is not strongly connected. As in $[\mathbf{2 5}]$ we write $\mathrm{SC}(G)$ for the set of strongly connected components of the graph $G$. Each such component defines a fractal within the vector of all fractals. For each $H \in \mathrm{SC}(G)$ we can determine the spectral exponent within the component $d_{\mathrm{s}}^{\mu}(H)$. There is a partial ordering such that $H_{1} \leqslant H_{2}$ if for $i \in H_{1}$, there is a directed path $\sigma \in E_{i}^{\infty}$ with $\sigma(m) \in H_{2}$ for some $m<\infty$. We can classify the strongly connected components, which correspond to classes in the classification of reducible matrices, into basic classes if the component has the same spectral exponent as the whole set. As in our discussion of reducible matrices, we write $S_{m}$ for the union of the classes of height $m+1$. Let $\tilde{H}_{j}=\{H \in \mathrm{SC}(G) \mid$ there is a path from $j$ to some vertex of $H\}$. We state the results for the Neumann counting function as exactly the same results (with different constants, periodic functions) hold for the Dirichlet counting function.

Theorem 5.6. For a general FRGD fractal the spectral exponent is defined by $d_{\mathrm{s}}^{\mu}=\max \left\{d_{\mathrm{s}}^{\mu}(H) \mid H \in \mathrm{SC}(G)\right\}$ and $d_{\mathrm{s}}^{\mu}\left(K_{i}\right)=\max \left\{d_{\mathrm{s}}^{\mu}(H) \mid H \in \tilde{H}_{i}\right\}$. If $i \in S_{0}$, then there is a periodic function $G_{i}$ such that

$$
\lim _{x \rightarrow \infty}\left(\frac{N_{i}(x)}{x^{d_{\mathrm{s}}^{\mu} / 2}}-G_{i}(\log x)\right)=0 .
$$

If $i \in S_{m}$, then there are constants $c_{7}(i)>0$ such that

$$
\lim _{x \rightarrow \infty} \frac{N_{i}(x)}{(\log x)^{m} x^{d_{s}^{\mu} / 2}}=c_{7}(i) .
$$

If $i \notin S$ and there is no path from $S$ to $i$, then

$$
\lim _{x \rightarrow \infty} \frac{N_{i}(x)}{x^{d_{\mathrm{s}}^{\mu} / 2}}=0 .
$$

If $i \notin S$ and there is a path from $S_{0}$ to $i$ but no path to $i$ from $S_{l}$, with $l>0$, then there is a periodic function $G_{i}^{\prime}$ such that

$$
\lim _{x \rightarrow \infty}\left(\frac{N_{i}(x)}{x^{d_{\mathrm{s}}^{\mu} / 2}}-G_{i}^{\prime}(\log x)\right)=0 .
$$

If $i \notin S$ and there is a path from $S_{m}$ to $i$ but no path to $i$ from $S_{l}$, with $l>m$, then there is a constant $c_{8}(i)>0$ such that

$$
\lim _{x \rightarrow \infty} \frac{N_{i}(x)}{(\log x)^{m} x^{d_{s}^{\mu} / 2}}=c_{8}(i) .
$$

Proof. We observe again that the Dirichlet counting function can be shifted and hence we can apply Corollary 2.12 to give the result.

Note that it would be possible to write out an explicit formula for the constants in terms of the function $\xi$ and the matrix of measures.

In the case where the limit is 0 , we can obtain a refinement by only considering the components feeding into that class and derive a corresponding eigenvalue counting-function result. We only state here the result for the case of the class of height 1 . Let the set of states which have access to a given component $H$ be denoted by $\mathcal{S}(H)$. 
Corollary 5.7. If $H$ is a component with $d_{\mathrm{s}}^{\mu}(H)<d_{\mathrm{s}}^{\mu}$, and if $i$ is in a class of height 1 in the $|\mathcal{S}(H)| \times|\mathcal{S}(H)|$-submatrix, then there is a periodic function $G_{H, i}$ such that

$$
\lim _{x \rightarrow \infty}\left(\frac{N_{i}(x)}{x^{d_{\mathrm{s}}^{\mu}(H) / 2}}-G_{H, i}(\log x)\right)=0 .
$$

\section{Examples}

\subsection{Plain mandala}

In [27] a fractal subset of $\mathbb{R}^{2}$, called plain mandala (inspired by the Buddhist image, mandala), was defined. Let $L$ denote the upper semicircle of unit radius with origin at the centre. Set $\xi_{1}=(-1,0), \xi_{2}=(1,0)$ and define for $i=1,2$ maps $F_{i}(x)=\frac{1}{2}\left(x+\xi_{i}\right)$. Also define $F_{3}, F_{4}: L \rightarrow L$ by

$$
F_{i}(x)=\phi\left(\frac{1}{2}\left(\psi(x)+\psi\left(\xi_{i-2}\right)\right)\right), \quad x \in K, \quad i=3,4,
$$

where $\phi:[0, \pi] \rightarrow L$ is defined by $\phi(\theta)=(\cos (\theta), \sin (\theta))$ and $\psi=\phi^{-1}$. The fractal is defined to be the set $K$ which satisfies

$$
K=\bigcup_{i=1,2} F_{i}(K) \cup L
$$

Though this cannot be directly expressed as a graph-directed fractal, as the maps $F_{3}$, $F_{4}$ are not similitudes, we can still use the above approach. The two types are given by the semicircle, $L$ and the 'mandala' $K$. Note that the semicircles map into themselves and into the mandalas but the mandalas only map to themselves and the graph describing the fractal will not be strongly connected. The graph-directed formulation for the fractal is

$$
\begin{aligned}
K & =\bigcup_{i=1,2} F_{i}(K) \cup \bigcup_{i=3,4} F_{i}(L), \\
L & =\bigcup_{i=3,4} F_{i}(L),
\end{aligned}
$$

and is illustrated in Figure 4. We can regard the construction graph as having two vertices (one for each type) and six edges (one for each map) as shown. We assign a resistivity $r_{j}$ to each edge of the graph which corresponds to map $F_{j}$. In the case where $r_{1}+r_{2}=1$ and $r_{3}+r_{4}=1$, and $t>1$, Murai [27] has shown the existence of a regular Dirichlet form with scaling:

$$
\begin{aligned}
& \mathcal{E}_{K}(f)=\sum_{i=1,2}\left(t r_{i}\right)^{-1} \mathcal{E}_{K}\left(f \circ F_{i}\right)+\sum_{i=3,4}\left(r_{i}\right)^{-1} \mathcal{E}_{L}\left(f \circ F_{i}\right), \\
& \mathcal{E}_{L}(f)=\sum_{i=3,4}\left(r_{i}\right)^{-1} \mathcal{E}_{L}\left(f \circ F_{i}\right) .
\end{aligned}
$$

In order to discuss the asymptotic scaling of the eigenvalues we choose a measure. As in $[\mathbf{2 7}]$, we take $\tau, s_{i}, i=1,2,3,4$, to be positive real numbers with $\tau \geqslant t, s_{1}+s_{2}=1$, 

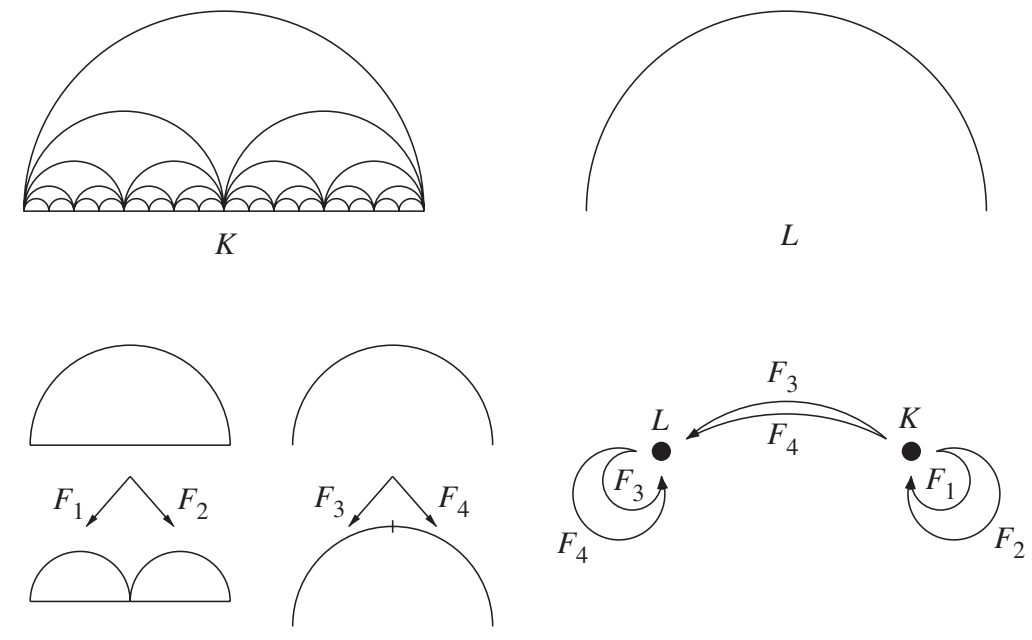

Figure 4. Plain mandala as an FRGD fractal.

$s_{3}+s_{4}=1, s_{1} s_{2}=s_{3} s_{4}$. Let $\left(\mu_{K}, \mu_{L}\right)$ be probability measures on $(K, L)$ defined as in (4.1) by the measures on the edges $\mu_{j}^{\prime}=\tau s_{j}, j=1,2$ and $\mu_{j}^{\prime}=s_{j}, j=3,4$.

As there are two strongly connected components we can compute the spectral exponent within each one. Let $d_{\mathrm{s}}^{1}, d_{\mathrm{s}}^{2}$ satisfy

$$
\sum_{i=1,2}\left(t r_{i} \tau s_{i}\right)^{d_{\mathrm{s}}^{1} / 2}=1, \quad \sum_{i=3,4}\left(r_{i} s_{i}\right)^{d_{\mathrm{s}}^{2} / 2}=1 .
$$

In $[\mathbf{2 7}]$ a result similar to Theorem 5.3 was established showing that the scaling in the spectral counting function could require a logarithmic correction factor, but the existence of the normalized limit was not established. In order to apply our main theorem we let the matrix renewal measure be

$$
M=\left[\begin{array}{cc}
\sum_{i=1}^{2}\left(t r_{i} \tau s_{i}\right)^{d_{\mathrm{s}}^{1} / 2} \delta_{-\log t r_{i} \tau s_{i}} & 0 \\
\sum_{i=3}^{4}\left(r_{i} s_{i}\right)^{d_{\mathrm{s}}^{2} / 2} \delta_{-\log r_{i} s_{i}} & \sum_{i=3}^{4}\left(r_{i} s_{i}\right)^{d_{\mathrm{s}}^{2} / 2} \delta_{-\log r_{i} s_{i}}
\end{array}\right] .
$$

The lattice case occurs for state $K$ if $\log \left(t r_{1} \tau s_{1}\right) / \log \left(t r_{2} \tau s_{2}\right) \in \mathbb{Q}$ and for state $L$ if $\log \left(r_{3} s_{3}\right) / \log \left(r_{4} s_{4}\right) \in \mathbb{Q}$. Thus, writing $N_{K}, N_{L}$ for either the Dirichlet or Neumann eigenvalue counting function associated with each type $K, L$, by Theorem 5.6 we have the following.

(1) If $d_{\mathrm{s}}^{1}=d_{\mathrm{s}}^{2}$, then there exist constants $c_{9}(i), i=K, L$, such that

$$
\lim _{\lambda \rightarrow \infty} \frac{N_{i}(\lambda)}{\lambda^{d_{\mathrm{s}}^{1} / 2} \log \lambda}=c_{9}(i), \quad i=K, L .
$$




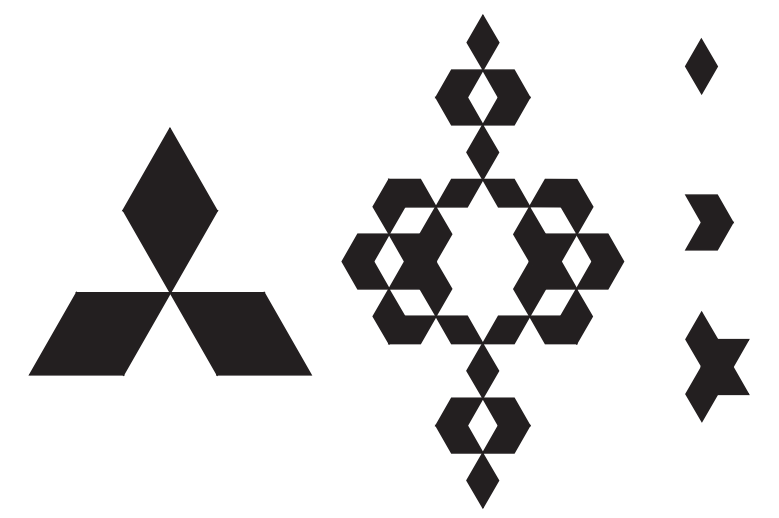

Figure 5. On the left is the generator of the diamond fractal; in the centre the diamond component at level 2 is shown; and on the right are the three component types - the diamond, the chevron and the crown.

(2) If $d_{\mathrm{s}}^{1}>d_{\mathrm{s}}^{2}$, then $d_{\mathrm{s}}=d_{\mathrm{s}}^{1}$ and there exist periodic functions $p_{K}, p_{L}$ such that

$$
N_{K}(\lambda)=\lambda^{d_{s} / 2}\left(p_{K}(\log \lambda)+o(1)\right)
$$

and

$$
\lim _{\lambda \rightarrow \infty} \frac{N_{L}(\lambda)}{\lambda^{d_{s} / 2}}=0
$$

but

$$
N_{K}(\lambda)=\lambda^{d_{s}^{2} / 2}\left(p_{L}(\log \lambda)+o(1)\right) .
$$

If state $i$ is in the non-lattice case, then $p_{i}$ is constant for $i=K, L$.

(3) For $d_{\mathrm{s}}^{2}>d_{\mathrm{s}}^{1}$, we set $d_{\mathrm{s}}=d_{\mathrm{s}}^{2}$ and there exist periodic functions $p_{i}^{\prime}$ such that

$$
N_{i}(\lambda)=\lambda^{d_{\mathrm{s}} / 2}\left(p_{i}^{\prime}(\log \lambda)+o(1)\right), \quad i=K, L .
$$

If state $i$ is in the non-lattice case, then $p_{i}$ is constant for $i=K, L$.

Remark 6.1. We do not have a proof that the periodic function is actually nonconstant in the lattice case.

\subsection{Fractals with infinitely ramified generator}

In this section we discuss a set of fractals in two dimensions whose generators are not finitely ramified but for which we can use graph-directed constructions. We will show the existence of a non-degenerate Dirichlet form on an example fractal from this class in the next subsection. It is an open problem whether the construction of such a form is possible in general.

Our starting point will be a regular polygon $P$ with edge set $E_{P}$ and vertex set $V_{P}$. Let $n_{P}=\left|V_{P}\right|$. We will let $\Theta_{C}$ be the rotation of $P$ sending $x \in V_{P}$ to its clockwise nearest neighbour. For $x, y \in V_{P}$, let $\Theta_{x y}$ be the mirror symmetry of $P$ that switches $x$ and $y$. 
Let $G$ be the symmetry group of $P$. For any line $L$, let $\Theta_{L}$ be the reflection in $L$. The $\Theta_{L}$ are not necessarily in $G$. For a pair of points $x, y$, let $L_{x y}$ be the line segment from $x$ to $y$. Example fractals that we consider here can be found in Figures 3 and 5 .

Definition 6.2. A set $A \neq \emptyset$ is finitely ramified relative to $B$ if $B$ is a proper superset of $A$, and $\left|\bar{A} \cap \overline{A^{c}}\right|$ is finite, where the complement is taken with respect to $B$.

Definition 6.3. Let $P$ be a regular polygon, and consider a set of affine maps $\left\{\phi_{i}\right\}_{i \in I}$, where $I$ is finite. For a subset $J \subset I$, define $\phi_{J}(\cdot)=\bigcup_{i \in J} \phi_{i}(\cdot)$ and $\Phi(\cdot)=\phi_{I}(\cdot)$. Assume the following.

(1) For all $i \in I, \phi_{i}(P) \subset P$.

(2) For all $\Theta \in G$, there is a map $\Theta_{I}: I \rightarrow I$ such that for all $i \in I, \Theta\left(\phi_{i}(P)\right)=$ $\phi_{\Theta_{I}(i)}(P)$. In particular, $\Theta(\Phi(P))=\Phi(P)$.

(3) For an $L \in E_{P}$, for all $i \in I$ such that $\phi_{I}(P) \cap L \neq \emptyset$ there exists a set $A_{i}$ with the finite ramification property relative to $\Phi(P)$ and an edge $L^{\prime} \in E_{P}$ such that $A_{i} \cap L^{\prime}=\emptyset$.

(4) For all $i, j \in I, i \neq j$, either

(i) $\phi_{i}(P) \cap \phi_{j}(P)=\emptyset$;

(ii) there are $x, y \in V_{P}$ such that $\phi_{i}(P) \cap \phi_{j}(P)=\phi_{i}(x)=\phi_{j}(y)$;

(iii) there are $L, L^{\prime} \in E_{P}$ such that $\phi_{i}(P) \cap \phi_{j}(P)=\phi_{i}(L)=\phi_{j}\left(L^{\prime}\right)$ (note that $\left.\Theta_{L} \circ \phi_{i}(P)=\phi_{j}(P)\right)$.

Let $K$ be the unique fixed point of $\Phi . K$ is then called a decomposable nested fractal.

Observation 6.4. A decomposable nested fractal $K$ is preserved under the symmetries of $P$, and $K \subset P$. Indeed, $K=\bigcap_{n=1}^{\infty} \Phi^{n}(P)$.

We will keep a parallel indexing system, so that if $X_{I}$ is a collection in $I, X_{Y}$ will be $\left\{\phi_{J}(Y): J \in X_{I}\right\}$. Typically, $Y$ will be $P$ or $K$.

For a decomposable nested fractal as above, let $\mathcal{A}_{I}$ be the collection of sets $J \subset I$ such that $\phi_{J}(P)$ has the finite ramification property relative to $\Phi(P)$. Then $\overline{\mathcal{A}}_{I}=\mathcal{A}_{I} \cup\{\emptyset, P\}$ is a $G$-invariant algebra. Let $\mathcal{B}_{I}=\left\{A \in \mathcal{A}_{I}: \neg \exists B \in \mathcal{A}_{I}[B \subset A]\right\}$. $\mathcal{B}_{I}$ is then $G$-invariant also. Let $\mathcal{A}_{P}, \mathcal{A}_{K}, \mathcal{B}_{P}$ and $\mathcal{B}_{K}$ be defined accordingly.

Definition 6.5. For a set $A$, let $B_{\varepsilon}(A)=\{x \in P: d(x, A)<\varepsilon\} . B_{\varepsilon}(x)=B_{\varepsilon}(\{x\})$.

Lemma 6.6. If $A \in \mathcal{B}_{P}$ and $x, y \in A$ are such that $B_{\varepsilon}(x)$ and $B_{\varepsilon}(y)$ are contained in $A$ for some $\varepsilon>0$, there is a curve $C$ and a $\beta>0$ such that $x, y \in B_{\beta}(C) \subset A$.

Proof. We easily see that there must be a sequence $S=\left\{s_{1}, \ldots, s_{n}\right\}$ such that $x \in$ $\phi_{s_{1}}(P)$ and $y \in \phi_{s_{n}}(P)$ and each pair $\phi_{s_{i}}(P)$ and $\phi_{s_{i+1}}(P)$ intersect at a line $\phi_{i}(L)$ for some $L \in E_{P}$.

Build $C$ piecewise by letting $C^{0}$ be the straight line from $x$ to the centre of $\phi_{s_{1}}(P)$, letting $C^{i}$ be the straight line from the centre of $\phi_{s_{i}}(P)$ to the centre of $\phi_{s_{i+1}}(P)$ for 
$i=1, \ldots, n-1$, and letting $C^{n}$ be the straight line from the centre of $\phi_{s_{i}}(P)$ to $y$. We have $C_{\varepsilon}^{0}, C_{\varepsilon}^{n} \subset A$, and $C_{l \cdot r_{\min }}^{n} \subset A$, so let $C=\bigcup_{i=0}^{n} C^{i}$ and $\beta=\varepsilon \wedge\left(l \cdot r_{\min }\right)$. Thus $B_{\beta}(C) \subset A$ as required.

Lemma 6.7. Let $x \in V_{P}$. If $K$ is connected, there is an $i \in I$ such that $x \in \phi_{i}(P)$.

Proof. By symmetry, there is either an $i \in I$ for each $x \in V_{P}$ so that $x \in$ $\phi_{i}(P)$, or there does not exist such an $i \in I$ for any $x \in V_{P}$. So assume such an $i \in I$ does not exist. Then $\Phi(P) \cap V_{P}=\emptyset$. By Observation 6.4 we get that $\phi_{A}(K) \cap \phi_{A^{c}}(K) \subset \phi_{A}(\phi(P)) \cap \phi_{A^{c}}(\phi(P))$. But at the same time, Definitions $6.3(3)$ and 6.3 (4) give us that $\phi_{A}(K) \cap \phi_{A^{c}}(K) \subset \phi_{A}\left(V_{P}\right) \cap \phi_{A^{c}}\left(V_{P}\right)$. These two together with $\Phi(P) \cap V_{P}=\emptyset$ give us that $\phi_{A}(K) \cap \phi_{A^{c}}(\boldsymbol{K})=\emptyset$, contrary to the assumption that $K$ is connected.

Observation 6.8. For $n_{P} \geqslant 4$, let $C$ be a curve in $P$ connecting two points $x, y$, belonging to two non-adjacent edges $L_{1}, L_{2} \in E_{P}$, respectively. By elementary topology, $C \cap \Theta_{C}(C) \neq \emptyset$.

Proposition 6.9. If $B \subset \mathcal{B}_{P}$ intersects with two lines $L_{1}, L_{2} \in E_{P}$, then $L_{1}$ and $L_{2}$ must be adjacent.

Proof. This is clearly true for $n_{P}=3$. For $n_{P}>3$, assume $L_{1}$ and $L_{2}$ are nonadjacent. Choose $\varepsilon>0, x \in B \cap L_{1}$ and $y \in B \cap L_{2}$ such that $B_{\varepsilon}(\{x, y\}) \subset B$. By Lemma 6.6, there is a curve $C$ connecting $x$ and $y$ and a $\beta>0$ such that $B_{\beta}(C) \subset B$. By Observation 6.8, there is a $z \in C \cap \Theta_{C}(C)$, and $B_{\varepsilon}(z) \in B \cap \Theta_{C}(B)$. This means there must be an $i \in I$ such that $\phi_{i}(P) \subset B \cap \Theta_{C}(B)$, and so $B_{I} \cap \Theta_{C}\left(B_{I}\right)$ is non-empty, and therefore in $\mathcal{A}_{I}$. This contradicts the assumption that $B \in \mathcal{B}_{P}$, unless $B=\Theta_{C}(B)$. But if $B=\Theta_{C}(B)$, then Definition 6.3 (3) for decomposable nested fractals gives us an $A_{i}$ such that $B \cap A$ is a proper subset of $B$ in $\mathcal{A}$, again contradicting $B \in \mathcal{B}$.

Corollary 6.10. $A$ set $B \subset \mathcal{B}_{P}$ intersects at most two lines $L_{1}, L_{2} \in E_{P}$.

Proof. Assume that $B$ intersects three or more lines in $E_{P}$. If $n_{P}=3, B$ intersects all $L \in E_{P}$, contradicting Definition 6.3 (3) for decomposable nested fractals. If $n_{P} \geqslant 4$, at least two of the lines will be non-adjacent, contradicting Proposition 6.9.

Corollary 6.11. If $B \in \mathcal{B}$ and $B$ intersects $L_{x y}$ on both sides of $z=\frac{1}{2}(x+y)$, then $B=\Theta_{x y}(B)$, and $B$ intersects no $L \in E_{P}, L \neq L_{x y}$.

Proof. To prove $B=\Theta_{x y}(B)$, we notice that if it was not, then $B \cap \Theta_{x y}(B)$ would be a proper subset of $B$ and at the same time an element of $\mathcal{A}$, contrary to the assumption that $B \in \mathcal{B}$. From this it follows that if $B$ intersects with an edge $L \neq L_{x y}$, it must also intersect $L^{\prime}=\Theta_{x y}(L)$, contradicting Corollary 6.10.

Let $\angle_{x}=L_{x y^{\prime}} \cup L_{x z^{\prime}}$, where $y^{\prime}=\frac{1}{2}(x+y), z^{\prime}=\frac{1}{2}(x+z)$, and $y, z$ are $x$ 's nearest neighbours in $V_{P}$. Let $\alpha$ be the angle in $\angle_{x}$. Further let $E=\bigcup_{L \in E_{P}} L$. 
Corollary 6.12. There is, for each $x \in V_{P}$, a $\Theta_{y z}$-symmetric $B \in \mathcal{B}$ such that $x \in B$ and $B \cap E=B \cap \angle_{x}$.

Fix $x$, set $P_{1}=\Phi(P)$ and let $P_{n}$ be the union of $\Phi(P)$ and a clockwise rotation by $\alpha$ degrees of $P_{n-1}$ around $x . P_{1}=\Phi(P)$. We define such $P_{n}$ for all $n$ smaller than or equal to the maximum number of rotations $360 / \alpha$. Let $\mathcal{B}_{n}$ be the corresponding smallest components, and let $\mathcal{C}^{\prime}=\cup_{n} \mathcal{B}_{n}$. Define an equivalence relation ' $\sim$ ' on $\mathcal{C}^{\prime}$ by letting $A \sim B$ if and only if there is a bijection $f: A \rightarrow B$ such that $d(x, y)=c \cdot d(f(x), f(y))$. Let $\mathcal{C}=\mathcal{C}^{\prime} / \sim$, and let $\mathcal{C}_{n}=\mathcal{B}_{n} / \sim$.

We want to see how $\mathcal{C}$ differs from $\mathcal{C}_{1}$; which new element types $(\mathcal{C})$ have we added from the old types $\left(\mathcal{C}_{1}\right)$ by the procedure above? It is clear that all of the new elements must be unions of old elements. Unions of elements can, again, only happen if two or more old-type elements have intersections of infinite cardinality. This can, again, only happen with $\mathcal{C}_{1}$-elements intersecting $E$. Let us then divide the old-type elements of $\mathcal{C}_{1}$ into three disjoint classes: $\mathcal{C}_{\mathrm{A}}$, elements which do not intersect with $E ; \mathcal{C}_{\mathrm{B}}$, elements which intersect exactly one $L \in E_{P}$; and $\mathcal{C}_{\mathrm{C}}$, elements which intersect exactly two $L \in E_{P}$. The previous results describe these sets, and guarantee that $\mathcal{C}_{\mathrm{C}}$ is non-empty.

Proposition 6.13. The types in the set $\mathcal{C}$ are in one-to-one correspondence with the elements of $\operatorname{FRGD}(K)$.

Proof. We need only show that each of the equivalence classes of $\Psi^{2}(P)$ is in oneto-one relation with $\mathcal{C}$. This, however, is now quite easy. We can divide the elements of $\Psi^{2}(P)$ into three classes. Firstly, those that are images, by some $\phi_{i}$, of an element of $\mathcal{C}_{\mathrm{A}}$ will correspond exactly to an element of $\mathcal{C}_{\mathrm{A}}$. Then we have the elements that have been created by a union of two images, by maps $\phi_{i}$ and $\phi_{j}$, respectively, of elements of $\mathcal{C}_{\mathrm{B}}$. They will correspond to elements of $\mathcal{C}_{2}$. Finally, we have the unions of images of elements of $\mathcal{C}_{\mathrm{C}}$. They are easily seen to correspond to elements in some $\mathcal{C}_{k}$ for $k \geqslant 2$.

An example of a decomposable nested fractal, called the diamond fractal in [18], with its vector of types is shown in Figure 5.

\subsection{Existence results}

The key assumption in our construction of the Laplacian on the graph-directed fractals was that there exists a fixed point for the map $\mathcal{T}$. It is a difficult problem to determine the existence of fixed points for the map, even in the single-component case. For the class of nested fractals, $[\mathbf{2 4}]$ showed that a fixed point would exist by using the symmetry of these fractals. In [30] conditions are given which show the existence of a fixed point for more general fractals, but these are very difficult to check in general.

We will follow [24] and give an existence result for a particular subclass of graphdirected nested fractals which are highly symmetric. We assume that the vector $\boldsymbol{r}$ has $r_{e}=1$, for all $e \in E$. 
Let $\left\{\phi_{e}: e \in E\right\}$ denote a family of similitudes in $\mathbb{R}^{n}$ associated with the edges of a graph $G$. As before there exists a set of compact subsets $K_{i} \subset \mathbb{R}^{n}$ such that

$$
K_{i}=\bigcup_{j \in \mathcal{S}} \bigcup_{e \in E_{i j}} \phi_{e}\left(K_{j}\right) .
$$

We call $K$ a graph-directed nested fractal if it satisfies the following assumption.

\section{Assumption 6.14.}

(1) $K$ is a finitely ramified graph-directed fractal.

(2) If $x, y \in F_{i}^{(0)}$, then reflection in the hyperplane $H_{x y}=\{z:|z-x|=|z-y|\}$ maps $F_{i}^{(n)}$ to itself.

The second part of the assumption is strong as the symmetry condition is required for each component. With it we can apply the approach of Lindstrøm [24] (or see [1]) to show that the map on transition probabilities has a fixed point. Such a fixed point can be used to construct a fixed point for the $\mathcal{T}$ map.

The fractal which appears in Figure 3 is a graph-directed nested fractal and hence there will be a solution to the fixed-point problem for this set. For the fractal in Figure 2, which is not a graph-directed nested fractal, discussions with Dr V. Metz have shown that, by techniques developed in [26], a fixed point will exist. The existence of the fixed point for the fractal of Figure 5 has also been demonstrated by a direct calculation in [18] and indirectly in $[\mathbf{2 6}]$.

Acknowledgements. The work of B.M.H. was undertaken at BRIMS, HewlettPackard Research Laboratories, Bristol, with the aid of a Royal Society Industry Fellowship and also at the Isaac Newton Institute, Cambridge, during the programme 'Mathematics and Applications of Fractals'. S.O.G.N. was supported at the University of Edinburgh by EPSRC grant GR/L07239. B.M.H. thanks Takashi Kumagai for drawing his attention to the paper [27], Volker Metz for comments on an earlier version of this paper, and a diligent referee for many useful comments.

\section{References}

1. M. T. BARLOW, Diffusions on fractals, in Lectures on probability theory and statistics, École d'été de probabilités de St Flour XXV (Springer, 1998).

2. M. T. BARlow AND J. Kigami, Localized eigenfunctions of the Laplacian on p.c.f. selfsimilar sets, J. Lond. Math. Soc. 56 (1997), 320-332.

3. M. F. BARnsley, J. H. Elton and D. P. Hardin, Recurrent iterated function systems, Constr. Approx. 5 (1989), 3-31.

4. J. CROFT, Some generalisations of nested fractal constructions and associated diffusions, PhD thesis, University of Warwick (1999).

5. F. M. Dekking, Recurrent sets, Adv. Math. 44 (1982), 78-104.

6. S. Dube, Using fractal geometry for solving divide and conquer recurrences, J. Aust. Math. Soc. B 37 (1995), 145-171.

7. K. J. Falconer, On the Minkowski measurability of fractals, Proc. Am. Math. Soc. 123 (1995), 1115-1124. 
8. K. J. FALCONER, Techniques in fractal geometry (Wiley, 1997).

9. M. Fukushima, Dirichlet forms, diffusion processes and spectral dimensions for nested fractals, in Ideas and methods in mathematical analysis, stochastics and applications. In memory of R. Høegh-Krohn (ed. S. Albeverio, J. Fensted, H. Holden and T. Lindstrøm), vol. 1, pp. 151-161 (Cambridge Universtiy Press, 1992).

10. M. Fukushima And T. Shima, On a spectral analysis for the Sierpinski gasket, Potential Analysis 1 (1992), 1-35.

11. B. M. Hambly, On the asymptotics of the eigenvalue counting function for random recursive Sierpinski gaskets, Prob. Theory Relat. Fields 117 (2000), 221-247.

12. B. M. Hambly and T. Kumagai, Transition density estimates for diffusion processes on post critically finite self-similar fractals, Proc. Lond. Math. Soc. 78 (1999), 431-458.

13. J. Kigami, Harmonic calculus on P.C.F. self-similar sets, Trans. Am. Math. Soc. 335 (1993), 721-755.

14. J. Kigami, Harmonic calculus on limits of networks and its application to dendrites, $J$. Funct. Analysis 128 (1995), 48-86.

15. J. Kigami, Distributions of localized eigenvalues of Laplacians on post critically finite self-similar sets, J. Funct. Analysis 156 (1998), 170-198.

16. J. Kigami, Analysis on fractals (Cambridge University Press, 2001).

17. J. Kigami and M. L. Lapidus, Weyl's problem for the spectral distribution of the Laplacian on P.C.F. self-similar fractals, Commun. Math. Phys. 158 (1993), 93-125.

18. J. Kigami, R. Strichartz and K. C. Walker, Constructing a Laplacian on the diamond fractal, Experimental Math. 10 (2001), 437-448.

19. T. Kumagai, Regularity closedness and spectral dimensions of the Dirichlet forms on p.c.f. self-similar sets, J. Math. Kyoto Univ. 33 (1993), 765-786.

20. S. Kusuoka, Diffusion processes on nested fractals, in Statistical mechanics and fractals (ed. R. L. Dobrushin and S. Kusuoka), Lecture Notes in Mathematics, vol. 1567 (Springer, 1993).

21. M. L. LApidus, Vibrations of fractal drums, the Riemann hypothesis, waves in fractal media and the Weyl-Berry conjecture, in Ordinary and partial differential equations, vol. IV, Proc. 12th Int. Conf. on Theory of Ordinary and Partial Differential Equations, Dundee, 1992, Research Notes in Mathematics, pp. 126-209 (Longman, London, 1993).

22. K. S. LAU, J. WAng And C. H. Chu, Vector valued Choquet-Deny theorem, renewal equation and self-similar measures, Studia Math. 117 (1995), 1-28.

23. M. LEVITIN AND D. VASSILIEV, Spectral asymptotics, renewal theorem and the Berry conjecture for a class of fractals, Proc. Lond. Math. Soc. 72 (1996), 188-214.

24. T. Lindstrøm, Brownian motion on nested fractals, Memoirs of the American Mathematical Society, vol. 83, no. 420 (1990).

25. R. D. MAuldin AND S. C. Williams, Hausdorff dimension in graph directed constructions, Trans. Am. Math. Soc. 309 (1988), 811-829.

26. V. Metz, 'Laplacians' on finitely ramified, graph directed fractals, preprint (2002).

27. J. MurAi, Diffusion processes on mandala, Osaka J. Math. 32 (1995), 887-917.

28. L. OLSEN, Multifractal spectrum for graph directed random recursive self-similar measures (Longman, London, 1996).

29. T. A. Ryan, A multidimensional renewal theorem, Ann. Prob. 4 (1976), 656-661.

30. C. SАвот, Existence and uniqueness of diffusions on finitely ramified self-similar fractals, Annls Sci. Ec. Norm. Super. 30 (1997), 605-673.

31. J. L. WANG, The open set condition for graph directed self-similar sets. Random Comput. Dynam. 5 (1997), 283-305. 